\title{
Fluorescence lifetime imaging - techniques and applications
}

\author{
W. BECKER \\ Becker \& Hickl GmbH, Nahmitzer Damm 30, 12277 Berlin, Germany
}

Key words. FLIM, FRET, TCSPC.

\section{Summary}

Fluorescence lifetime imaging (FLIM) uses the fact that the fluorescence lifetime of a fluorophore depends on its molecular environment but not on its concentration. Molecular effects in a sample can therefore be investigated independently of the variable, and usually unknown concentration of the fluorophore. There is a variety of technical solutions of lifetime imaging in microscopy. The technical part of this paper focuses on time-domain FLIM by multidimensional time-correlated single photon counting, time-domain FLIM by gated image intensifiers, frequency-domain FLIM by gainmodulated image intensifiers, and frequency-domain FLIM by gain-modulated photomultipliers. The application part describes the most frequent FLIM applications: Measurement of molecular environment parameters, protein-interaction measurements by Förster resonance energy transfer (FRET), and measurements of the metabolic state of cells and tissue via their autofluorescence. Measurements of local environment parameters are based on lifetime changes induced by fluorescence quenching or conformation changes of the fluorophores. The advantage over intensity-based measurements is that no special ratiometric fluorophores are needed. Therefore, a much wider selection of fluorescence markers can be used, and a wider range of cell parameters is accessible. FLIM-FRET measures the change in the decay function of the FRET donor on interaction with an acceptor. FLIM-based FRET measurement does not have to cope with problems like donor bleedthrough or directly excited acceptor fluorescence. This relaxes the requirements to the absorption and emission spectra of the donors and acceptors used. Moreover, FLIM-FRET measurements are able to distinguish interacting and noninteracting fractions of the donor, and thus obtain independent information about distances and interacting and noninteracting protein fractions. This is information not accessible by steady-state FRET techniques. Autofluorescence FLIM exploits changes in the decay parameters of endogenous fluorophores with the

Correspondence to: Wolfgang Becker, Becker \& Hickl GmbH, Nahmitzer Damm 30, 12277 Berlin, Germany. metabolic state of the cells or the tissue. By resolving changes in the binding, conformation, and composition of biologically relevant compounds FLIM delivers information not accessible by steady-state fluorescence techniques.

\section{Introduction}

Fluorescence techniques have found broad application in microscopy of live specimens because they are extremely sensitive and deliver information about biochemical interactions on the molecular scale. Apart from the fact that samples may be labelled with fluorophores, fluorescence techniques are noninvasive and nondestructive, and thus can be applied to live specimens. The development of fluorescence techniques has especially benefited from the introduction of multidimensional microscopy techniques. Data are obtained over three spatial dimensions, over the wavelength, the experiment time and the polarisation of the light. However, fluorescence is not only characterised by the spatial distribution of the fluorescence intensity and the fluorescence spectrum. There is also a characteristic fluorescence decay function: When a molecule absorbs a photon it enters an excited state. From this state it can return to the ground state by emitting a photon, by internally converting the absorbed energy into heat, by passing the energy to its molecular environment, or by crossing into the triplet state and return to the ground state from there (Lakowicz, 2006). For a homogenous population of molecules the resulting fluorescence decay is a single exponential function. The time constant of this function, the fluorescence lifetime, is the reciprocal sum of the rate constants of all possible return paths. The rate constants, and thus the fluorescence lifetime, depend on the type of the molecule, its conformation and on the way the molecule interacts with its environment.

Of all fluorescence parameters, it is the fluorescence decay function that yields the most direct insight into the molecular interactions of a fluorophore with its biological environment. The fluorescence decay function is obtained if the fluorescence is excited by short laser pulses, and the fluorescence intensity after the pulses is measured at a resolution on the picosecond or nanosecond time scale. 


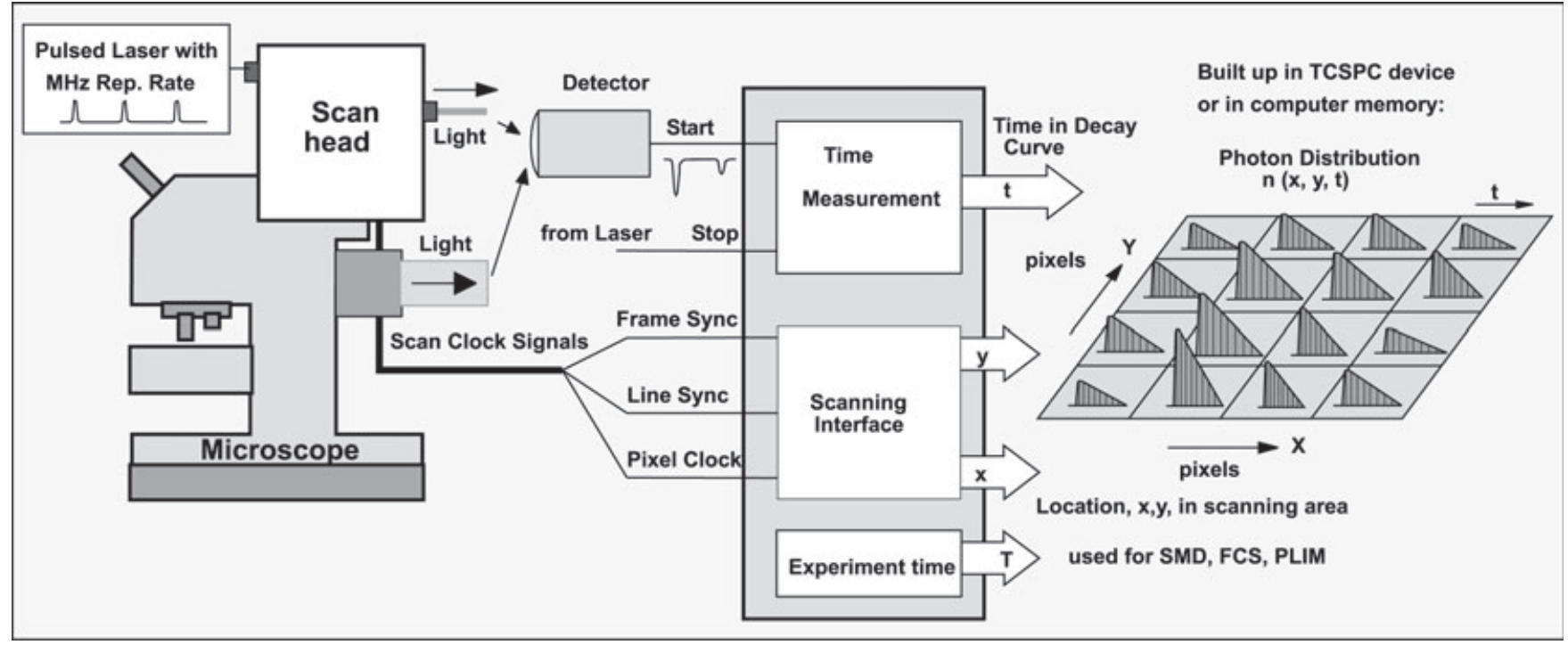

Fig. 1. Principle of TCSPC FLIM.

\section{FLIM techniques}

Fluorescence lifetime imaging (FLIM) techniques can be classified into time-domain and frequency-domain techniques, photon counting and analog techniques and point-scanning and wide-field imaging techniques. It also matters whether a technique acquires the signal waveform in a few time gates (Buurman et al., 1992) or in a large number of time channels, and whether this happens simultaneously (Becker, 2005) or sequentially (Dowlinget al., 1997; Straub \& Hell, 1998). Virtually all combinations are in use. This leads to a wide variety of instrumental principles. Different principles differ in their photon efficiency, i.e. in the number of photons required for a given lifetime accuracy (Ballew \& Demas, 1989; Köllner \& Wolfrum, 1992; Gerritsen et al., 2002; Philip \& Carlsson, 2003), the acquisition time required to record these photons, the photon flux they can be used at, their time resolution, their ability to resolve the parameters of multiexponential decay functions, multiwavelength capability, optical sectioning capability and compatibility with different imaging and microscopy techniques. An overview has been given by Becker \& Bergmann (2008).

\section{Time-domain FLIM by TCSPC}

Time correlated Single photon counting (TCSPC) FLIM uses a multidimensional time-correlated single photon counting process (Becker, 2005; Becker, 2010) which is an extension of the classic TCSPC technique (O'Connor \& Phillips, 1984). The principle is shown in Figure 1. The sample is scanned by the focused beam of a high-frequency pulsed laser. Data recording is based on detecting single photons of the fluorescence light, and determining the arrival times of the photons with respect to the laser pulses as well as the position of the laser beam in the moment of photon detection. From these parameters, a photon distribution over the spatial coordinates, $x, y$ and the times of the photons, $t$, after the laser pulses is built up. The result is a three-dimensional data array that represents the pixels of the two-dimensional scan, with each pixel containing photons in a large number of time channels for consecutive times after the excitation pulses.

Among the FLIM techniques described in this paper multidimensional TCSPC delivers the highest time resolution. It also delivers the best lifetime accuracy or photon efficiency, for a given number of photons detected from the sample (Ballew \& Demas, 1989; Köllner \& Wolfrum, 1992; Philip \& Carlsson, 2003). TCSPC FLIM has a number of other features important to lifetime imaging of biological systems: It is able to resolve complex decay profiles, and it is tolerant to dynamic changes in the fluorescence decay parameters during the acquisition (Becker, 2005; Becker, 2010). Moreover, TCSPC FLIM is perfectly compatible with confocal and multiphoton (Wilson \& Sheppard, 1984; Denk et al., 1990; Diaspro, 2001; Pawley, 2006) laser scanning microscopes. It has no problems with the fast scan rate used in these systems: The recording process is just continued over as many frames of the scan as necessary to obtain the desired signal-to-noise ratio. Moreover, TCSPC FLIM takes advantage of the optical sectioning capability of confocal or multiphoton scanning: The data are obtained from an accurately defined plane of the sample, without contamination by out-of-focus fluorescence.

More parameters can be added to the photon distribution, such as the wavelength of the photons, the wavelength of several multiplexed excitation lasers or the time from a periodic stimulation of the sample. The wavelength of the photons is obtained by splitting the fluorescence light spectrally and projecting the spectrum on the elements of a multi-anode 

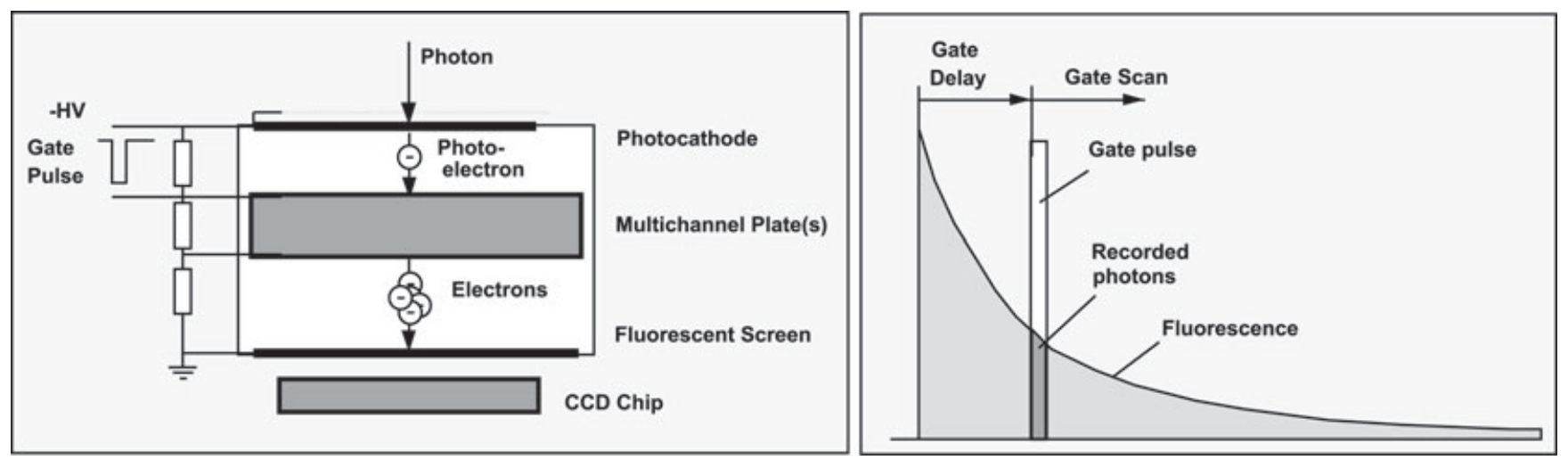

Fig. 2. Gated image intensifier.

photomultiplier tube (PMT). The FLIM systems determines the PMT channel (or wavelenght channel) number in which a particular photon was detected. The recording process builds up a photon distribution over the spatial coordinates, $x, y$, the times of the photons, $t$, and the wavelength channel, $\lambda$. The result is a data set that contains decay curves for several wavelengths in the particular pixels (Becker et al., 2002, 2007; Bird et al., 2004; Becker, 2005). Applications to FRET and to the photoconversion of photodynamic therapy (PDT) dyes have been described by Biskup et al. (2007) and Rück et al. (2007).

A similar process can be used to obtain lifetime images at different excitation wavelengths. Several excitation wavelengths are multiplexed either frame by frame, line by line or even within one pixel. The laser wavelength channel number is used as a coordinate of the photon distribution.

Fast dynamic processes can be recorded by using the time after a stimulation of the sample as an additional recording coordinate. A technique called 'fluorescence lifetime-transient scanning' uses line scanning to record lifetime changes down to a resolution of $1 \mathrm{~ms}$ (Becker et al., 2012).

In addition to building up FLIM data TCSPC makes the full information about the individual photons available. Such 'parameter tagged photon data' can be used in various ways, e.g. for single-molecule detection (Prummer et al., 2004; Widengren et al., 2006), fluorescence correlation spectroscopy, (Felekyan et al., 2005; Becker et al., 2006) and phosphorescence lifetime imaging, Becker et al., $2011 \mathrm{a}$.

As a disadvantage of TCSPC FLIM it is often stated that TCSPC FLIM is slow in terms of acquisition time. This is not quite correct, as has been shown by Becker et al. (2004b, 2009) and Katsoulidou et al. (2007). Certainly, a TCSPC FLIM system cannot record an image faster than the scanner is able to scan the sample. Also, the count rate a TCSPC system can process is limited (Becker, 2005). However, the count rates achieved in typical TCSPC FLIM applications are rather limited by the photostability of the samples than by the counting capability of the FLIM system. Due to its near-ideal photon efficiency,
TCSPC FLIM in these cases achieves the shortest acquisition time of all FLIM techniques based on sample scanning.

\section{Time-domain FLIM by gated image intensifiers}

Image intensifiers are vacuum tubes containing a photocathode, a multiplication system for the photoelectrons and a two-dimensional image detection system. Multichannel plates are used for electron multiplication, see Figure 2. One microchannel plate gives a typical multiplication factor of 1000; a gain of $10^{6}$ can be achieved by two plates in series.

Time resolution is obtained by gating the image intensifier. This is achieved by applying a gating pulse between the multichannel plate and the photocathode or a conductive grid capacitively coupled to it (Dymoke-Bradshaw, 1992). The fluorescence decay functions are measured by scanning the gate pulse in time, i.e. by taking consecutive images for different delay of the gate pulse, see Figure 2 (right).

Gated image intensifiers are normally used in combination with wide-field excitation (Wang et al., 1992; Scully et al., 1996; Dowling et al., 1997) or multiphoton multibeam scanning (Straub \& Hell, 1998). The advantage of widefield imaging is that the spatial information is acquired simultaneously for all pixels. Although the temporal information is acquired sequentially the acquisition times can be made shorter than for techniques based on spatial scanning.

The disadvantage is that gating records only a part of the photons. The photon efficiency of the recording is therefore low, and the total sample exposure is high. Moreover, widefield excitation does not reject out-of-focus light. Unless the samples are very thin the decay data are thus contaminated by fluorescence from out-of-focus planes. The result is not only a loss in image contrast but also an addition of unwanted lifetime components to the decay functions.

The efficiency problem can be partially solved by recording in only a few time gates. Images in these time gates are recorded either sequentially, or simultaneously by projecting several images of the object on the photocathode through different 


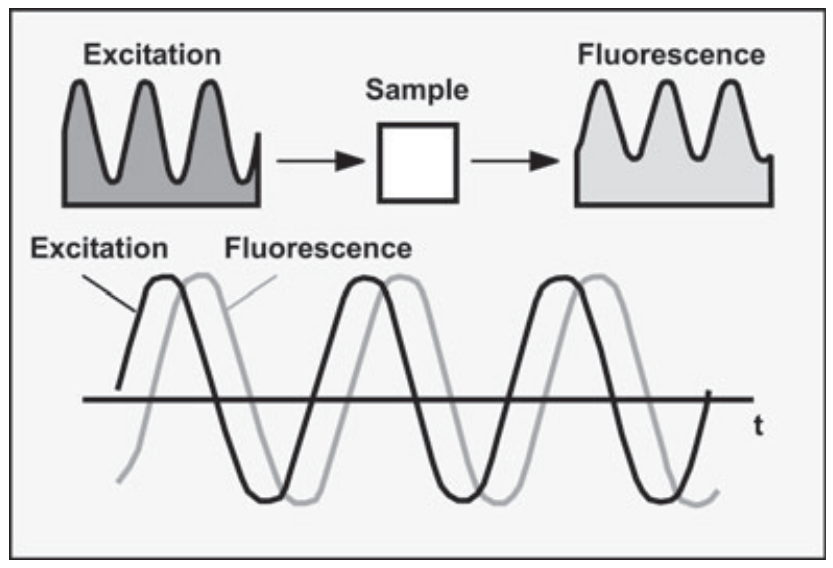

Fig. 3. Principle of frequency-domain FLIM. The fluorescence lifetime is derived from the decrease in the modulation degree and the phase shift of the fluorescence compared to the excitation.

optical delays (Agronskaia et al., 2003; Elson et al., 2004). The problem is that the decay functions are undersampled. It is therefore difficult to resolve multiexponential decay profiles into their lifetimes and amplitude coefficients.

Out-of-focus signals can, in principle, be removed from the data by structured illumination techniques (Cole et al., 2001). However, these techniques are based on calculating differences between images recorded for different illumination patterns. Although the result is an image containing only the signal from the focal plane this image contains the shot noise of the photons of all planes from which fluorescence is detected.

Systems based on gated image intensifiers can be used for multispectral FLIM. Images in different wavelength intervals are projected on the same image intensifier tube and recorded within one and the same gate scan. It is also possible to project a line of excitation light on the sample, and project the fluorescence light from this line on the entrance slit of a spectrograph. The sample is then scanned by either moving the sample or the excitation perpendicularly to the extension of the line. The spectra at the output of the spectrograph are recorded by the gated image intensifier. Because the excitation line and the spectrograph slit form a confocal system the technique obtains out-of-focus suppression. A system of this design was described by De Beule et al. (2007).

\section{Frequency-domain FLIM}

The time-domain and the frequency-domain are related via the Fourier transform. Time-domain data have intensity values in subsequent time channels. In the frequency-domain, these translate into amplitude and phase values at multiples of the signal repetition frequency. This however, does not mean that the recording principles and the instruments are exactly equivalent: There are time-domain techniques which record a full waveform directly into a large number of time channels. However, there is no technique that records the amplitude and phase values at all multiples of the modulation or pulse repetition frequency simultaneously. Most frequency-domain FLIM instruments therefore record amplitude and phase at the fundamental pulse repetition frequency only. These data are used directly to characterise the fluorescence decay behaviour of the sample, see section 'Data Analysis'.

The general principle is shown in Figure 3. The sample is excited by modulated or pulsed light. The fluorescence of the sample has a decreased modulation degree and a phase shift compared to the excitation. These values are measured and used to determine the fluorescence decay behaviour.

Detection of frequency-domain FLIM data is possible by wide-field-excitation and a gain-modulated camera (Lakowicz \& Berndt, 1991), or by scanning and detecting the fluorescence by gain-modulated point-detectors (Gratton \& Barbieri, 1986). The principle is shown in Figure 4. The gain of the camera or the detectors is modulated by an oscillator frequency, $f_{\text {osc }}$, slightly different from the laser pulse or modulation frequency, $f_{\text {laser }}$. The phase shift and the amplitude of the fluorescence signal then transfer into a signal at the
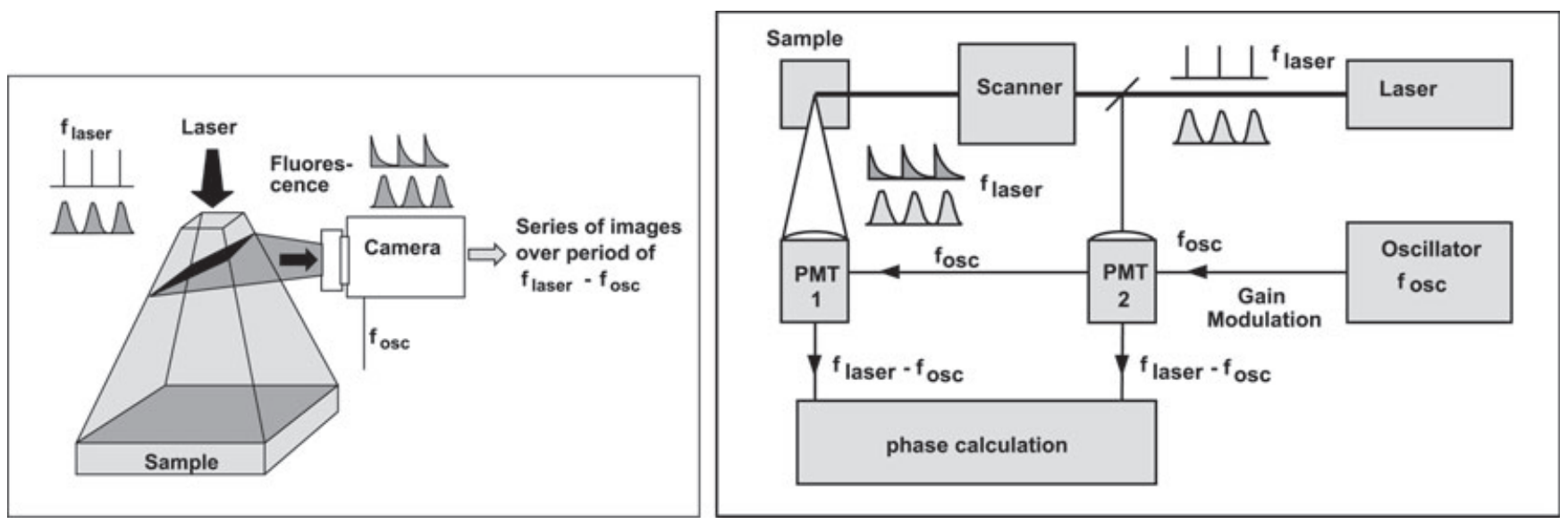

Fig. 4. Frequency-domain FLIM. Left: Modulated camera. Right: scanning and modulated point-detectors. 
difference frequency, $f_{\text {laser }}-f_{\text {osc }}$. This is the same 'heterodyne' principle as it is used in a radio. It has in fact been shown that a frequency-domain FLIM system can be built using low-cost telecommunication components (Booth \& Wilson, 2004). The advantage of the heterodyne principle is that the difference frequency can be made independent of and much lower than the laser frequency. The phase and the amplitude are determined at the difference frequency where filtering and signal processing can be performed by digital techniques.

Both the modulation of the excitation light and the modulation of the detectors need not necessarily be sinusoidal. Frequency-domain systems can therefore also be used with high-frequency pulsed lasers (Gratton \& Barbieri, 1986).

Frequency-domain FLIM treats the fluorescence signal as an analog waveform, not as individual photon detection events. It is therefore able to work at extremely high intensities, where single photon detection becomes impossible. Problems may occur at extremely low intensities, when the detector, on average, delivers less than one photon within the time interval of the phase calculation. What then happens depends on the details of the electronics used.

The photon efficiency of frequency-domain FLIM depends on a number of instrumental details. The efficiency increases with the modulation degree in the excitation light source and in the detectors. Ideal modulation would mean that both the excitation light intensity and the gain of the detectors had to be varied between $-100 \%$ and $+100 \%$. For sinusoidal excitation this is not possible because neither parameter can be reversed in polarity. The best efficiency is obtained by using short laser pulses, and by modulating the detectors by squarewave signals. Moreover, it matters whether the detectors are really modulated in their gain or rather in their photon detection efficiency. Efficiency modulation suppresses a part of the photons and results in a suboptimal signal-to-noise ratio. Details can be found in Philip \& Carlsson (2003).

An experimental comparison of TCSPCFLIM and frequencydomain FLIM by modulated point detectors has been given by Gratton et al. (2003). The authors found that the signalto-noise ratio of the lifetimes obtained for low-concentration samples and low count rates is indeed better for TCSPC FLIM. At high concentration (high count rates) the signal-to-noise ratio of both techniques converge.

\section{Other FLIM techniques}

There is a number of other FLIM techniques and combinations with microscopy techniques which can only be mentioned briefly here.

Time-gated photon counting is used in combination with laser scanning to record fluorescence lifetime images at high efficiency and at extremely high count rates (Buurmanet al., 1992). The technique counts the photons detected by a high-speed detector in several parallel gated counters. In principle, gated photon counting can handle extremely high count rates up to the overload limit of the detector. The tradeoff is that the number of time gates is very limited. The temporal data are thus undersampled, and the amplitudes and lifetimes parameters of multiexponential decay profiles are difficult to obtain.

Also streak cameras have been used to record FLIM in combination with laser scanning microscopes. The capability of the streak camera to resolve an optical signal both in $x$ and $t$ has been used to record multispectral FLIM data (Biskup et al., 2007) or to record a full line of the scan before the data are read out (Krishnan et al., 2003). The performance of such systems strongly depend on the parameters of the streak camera, especially the excitation pulse rate the camera can handle, and the speed the data can be read out.

TCSPC can be used in combination with position-sensitive detectors (Kemnitz, 2001). The result is a wide-field FLIM techniques with single-photon sensitivity and excellent time resolution. The drawback is that no depth resolution is obtained.

TCSPC (scanning) FLIM has been combined with multibeam scanning (Kumar et al., 2007). The light signals from several excited spots were projected on the cathode elements of a multi-anode PMT. A single TCSPC module was used to build up a photon distribution over the times of the photons in the fluorescence decay, the scan coordinates, and the detector channel of the multi-anode PMT. The principle can probably be made faster by using parallel TCSPC channels. The feasibility of an eight-channel parallel TCSPC system has been demonstrated for multispectral FLIM (Becker et al., 2009).

Directly gated (Mitchell et al., 2002a) and modulated (Mitchell et al., 2002b) charge-coupled device (CCD) image sensors have been used for wide-field FLIM at moderate time resolution. The technique intrigues by simplicity and low cost but is not yet fast enough to obtain FLIM data at subnanosecond or picosecond resolution.

Attempts to build image sensors based on an array of single-photon avalanche photodiodes are at an early stage of development. The array must be cooled to low temperature to keep the single-photon avalanche photodiodes working. The times of the photons are determined by integrating individual time-to-digital converters on the sensor chip for each pixel or each line. The problem of this approach is the dark count rate and the high readout speed required: A sensor of 100000 pixels would deliver an integral dark count rate on the order of $10^{6}$ photons s $^{-1}$. The signal count rate should be at least 10 to 100 times higher. Position and timing data require about 24 bits per photon. A data transfer rate of $240 \mathrm{Mb} \mathrm{s}^{-1}$ to $2.4 \mathrm{~Gb} \mathrm{~s}^{-1}$ would thus be required to read out the photon data. A transfer rate this high is difficult to reach but not impossible. However, the fast timing and data transfer electronics generates heat. It is thus difficult to keep the sensor at a sufficiently low temperature, which, in turn increases the dark count rate.

Lifetime imaging by an image intensifier followed by a fast-readout camera has been demonstrated by Petrasek \& 


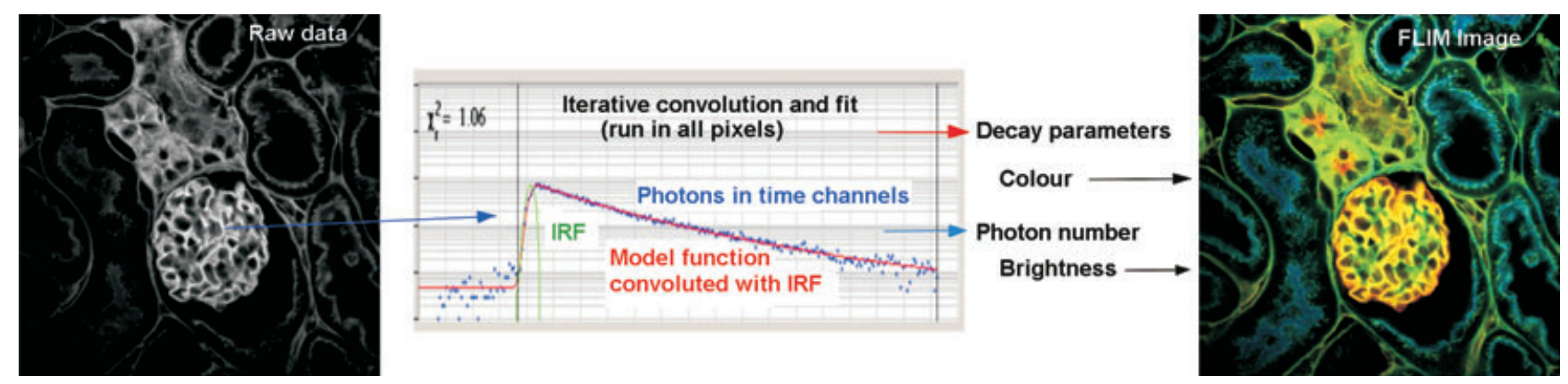

Fig. 5. Time-domain FLIM data analysis.

Suhling, 2010. By detecting single photons and defining the $x-y$ position by centroiding an excellent optical resolution of the camera system is obtained. Because no gating is used, the system records at high photon efficiency. At first glance, it may appear that the time resolution is limited by the frame rate of the camera $(250 \mathrm{kHz})$. However, the authors found a way to use the luminescence decay time of the fluorophore at the output of the image intensifier to identify the arrival times of the photons within the frames. The resulting time resolution was $40 \mathrm{~ns}$.

\section{Data analysis}

\section{Time-domain data}

Time-domain FLIM data (from TCSPC FLIM systems or gated camera systems) are arrays of pixels each of which contains a (usually large) number of time channels. The time channels contain photon numbers for consecutive times after the excitation pulse, see Figure 5(middle). To obtain fluorescence decay parameters from such data an iterative convolution process is used. The function of a suitable decay model is convoluted with the instrument response function (IRF). The IRF is the response of the detection system to the excitation pulse itself. It is either measured or calculated from the fluorescence decay data. The fit procedure then optimises the model parameters until the best fit to the photon numbers in the time channels is achieved. The principle is in use for analysis of single fluorescence decay curves for many years (O'Connor \& Phillips, 1984). For FLIM, the fit procedure has to be repeated for all pixels of the image.

The simplest decay model is a single exponential function. It is described by a single decay time. In most cases, however, the decay profiles have to be modelled by sums of two or three exponential functions. These models are described by several decay times and amplitude coefficients. The final FLIM image is obtained by assigning the brightness to the total photon number in the pixel, and the colour to a selected decay parameter (Fig. 5, right). The parameter can be the lifetime of a single-exponential decay, an amplitude- or intensity-weighted average of the lifetimes in a multiexponential decay, or a lifetime or amplitude ratio.

There are several modifications of the analysis algorithm. There methods that efficiently extract decay data from noisy decay curves, such as maximum likelihood analysis or the Bayesian analysis, please see Prummer et al. (2004) and Rowley et al. (2011). For low photon numbers a singleexponential lifetime can also be efficiently derived from the first moment (the average arrival time) of the photon distribution. The lifetime is the difference of the first moments of the decay data and the IRF. The first-moment calculation requires that the decay curves be recorded with negligible background and over a time interval containing the complete decay curves. Under these conditions the first-moment calculation delivers an ideal signal-to-noise ratio. The drawback is that it does not deliver parameters of multiexponential decay profiles.

Time-domain analysis has been extended by 'multiparameter' analysis (Weidkamp-Peters et al., 2009). This technique builds up two-dimensional histograms of pixel frequencies over several decay parameters obtained in one detection channel, or decay parameters and intensity ratios obtained in different detection channels. In these histograms, signatures of fluorophores or fluorophore fractions can be found, marked, and the corresponding pixel be backannotated in the images.

Frequency-domain data

Frequency-domain data contain two numbers in each pixel: A phase and a modulation degree. Such data can be analysed elegantly by the 'Phasor' approach (Digman et al., 2008). Phasor analysis does not explicitly aim on determining fluorescence lifetimes or decay components for the pixels. Instead, it uses the phase and the modulation degree directly. For each pixel, a pointer (the phasor) is defined and displayed in a polar plot. The phase is used as the angle of the pointer, the modulation degree as the amplitude. This 'phasor plot' has several remarkable features:

- The phasors of pixels with single-exponential decay profiles end on a semicircle. The angle of the phasor, i.e. the location on the semicircle, depends on the fluorescence lifetime. 


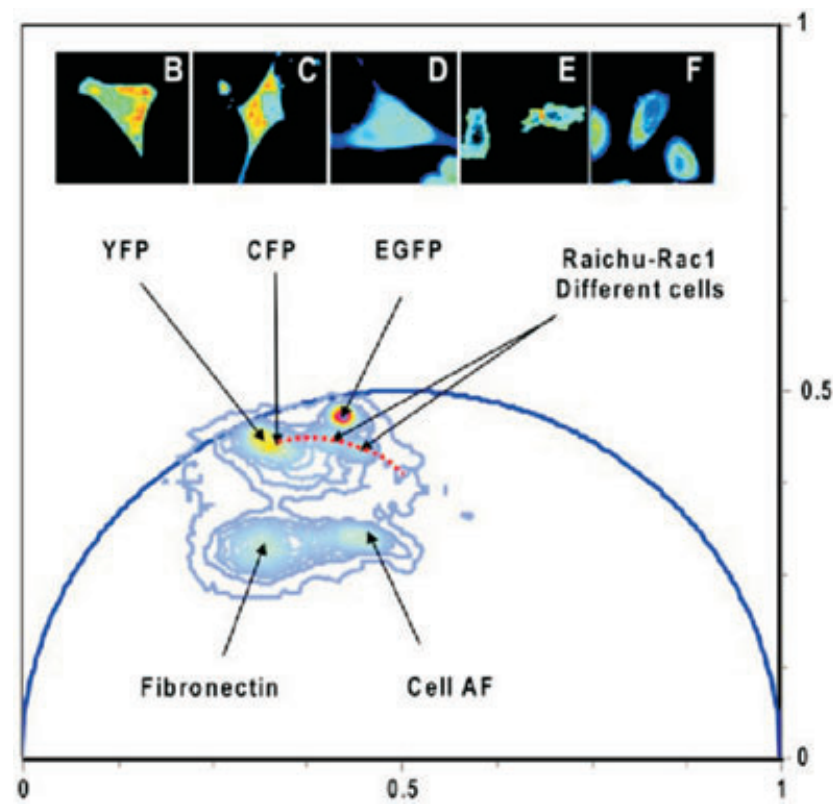

Fig. 6. Phasor analysis. Five different cells, transfected with (B) YFP, (C) paxillin-CFP, (D) EGFP, and (E) RAICHU-Rac1. (F) is an untransfected cell emitting only autofluorescence. From Digman et al. (2008).

- Phasors of pixels with multiexponential decay profiles, i.e. sums of several decay components, end inside the semicircle.

- Phasors of decay profiles which result from the convolution of several processes end outside the semicircle. An example is the emission of a FRET-excited acceptor, which is the convolution of the decay function of the interacting donor with the fluorescence decay of the acceptor.

Pixels with different signature in the phasor plot are then back-annotated in the image by giving them different colours. An example is shown in Figure 6. It shows a phasor plot for several cells, B to F, shown at the top of the figure. The cells were transfected with (B) yellow fluorescent protein (YFP), (C) paxillin-cyan fluorescent protein (CFP), (D) enhanced green fluorescent protein (EGFP) and (E) RAICHU-Rac1 (Graham et al., 2001). (F) is an untransfected cell. As can be seen in the lower part of the figure, the fluorophores form different spots in the phasor plot, as does the autofluorescence of the untransfected cells. Note that all spots are inside the semicircle, i.e. none of the fluorophores delivers a perfectly single-exponential decay in the cell environment.

Phasor analysis is not restricted to data obtained in frequency-domain systems. It can be applied to Fouriertransformed time-domain data as well (Digman et al., 2008).

\section{FLIM applications}

Why use the fluorescence lifetime?

Microscopists often consider the fluorescence lifetime just an additional parameter to separate the signals of different fluorophores. It is certainly correct that different fluorophores usually have different fluorescence lifetimes, and their signals thus can be separated by FLIM. However, discrimination of different fluorophores can be better and more easily achieved by spectrally resolved microscopy techniques.

Typical FLIM applications are therefore not primarily aiming at separating signals of different fluorophores. Instead, FLIM is used to discriminate different fractions of the same fluorophore in different states of interaction with its environment. This is achieved by using a basic property of the fluorescence lifetime: The fluorescence lifetime of a fluorophore depends on its molecular environment but, within reasonable limits, not on its concentration. Molecular effects can thus be investigated independently of the unknown and usually variable fluorophore concentration (Lakowicz, 2006; Berezin \& Achilefu, 2010; Roberts et al., 2011).

Effects used in FLIM applications are:

- Quenching of the fluorescence or phosphorescence by various ions, especially $\mathrm{Ca}^{2+}$ and $\mathrm{Cl}^{-}$, both of which are important to the function of the neuronal system (Kaneko et al., 2004; Gilbert et al., 2007; Funk et al., 2008; Kuchibhotla et al., 2009).

- Fluorescence or phosphorescence quenching by oxygen. Oxygen is an efficient fluorescence quencher for a large number of fluorophores (Lakowicz, 2006), especially those of longer fluorescence lifetime. Strong quenching by oxygen is observed for the phosphorescence of organic complexes of ruthenium, europium, platinum and palladium (Lebedev et al., 2009).

- A strong oxygen effect exists on the fluorescence of the endogenous fluorophores nicotinamide adenine dinucleotide (NADH) and flavin adenine nucleotide (FAD). The effect has been found already in the 1930s. Chance et al. defined a 'redox ratio' that is a direct indicator of the amount of oxygen used in the mitochondria of the cells (Chance, 1976; Chance et al., 1979). Effects of oxygen probably exist also for other endogenous fluorophores (Schweitzer et al., 2004).

- Fluorescence lifetimes change on binding of a fluorophore to a biological target. The most likely mechanism is a change in the conformation of the fluorophore which, in turn, changes the rate of internal nonradiative decay. For almost all dyes used in cell biology the fluorescence lifetime depends more or less on the binding to proteins, DNA or RNA (Van Zandvoort et al., 2002), and on the conformation of the protein they are bound to (Treanor et al., 2005; Benesch et al., 2007). Also NADH and FAD change their lifetimes on binding to proteins. Both the fluorescence lifetimes and the relative amplitudes of the decay components have been found sensitive to the metabolic state of cells and tissue.

- $\mathrm{pH}$ : Many fluorescent molecules have a protonated and a deprotonated form. The equilibrium between both depends on the $\mathrm{pH}$. If the protonated and deprotonated forms have 
different lifetimes the apparent lifetime is an indicator of the pH. (Sanders et al., 1995; Hanson et al., 2002).

- Förster Resonance Energy Transfer: Förster resonance energy transfer (Förster, 1946; 1948, see also Förster, 2012, translation by Klaus Suhling), or FRET, is an interaction in which energy from the first molecule, the donor, transfers into the second one, the acceptor. FRET is used to investigate protein interactions and protein configuration. The measurement of FRET is the most frequent FLIM application.

- Aggregates: The radiative and nonradiative decay rates depend on possible aggregation of the dye molecules. Aggregation is influenced by the local environment; the associated lifetime change can be used as a probe function. Aggregation has been used to observe the internalisation of dyes into cells (Kelbauskas \& Dietel, 2002).

- Local viscosity: Viscosity can be sensed by 'molecular rotors'. These are fluorophores that have a high degree of internal flexibility. Rotation inside the fluorophore provides for a radiationless decay path. The nonradiative decay rate changes with the viscosity of the solvent. Viscosity measurement by FLIM with a molecular rotor has been demonstrated by Kuimova et al. (2008) and Levitt et al. (2009a).

- Proximity to metal surfaces: Extremely strong effects on the decay rates can be expected if fluorophores are bound to metal surfaces, especially to metallic nano-particles (Malicka et al., 2003; Ritman-Meer et al., 2007; Cade et al., 2010). Similar, yet less dramatic effects have been reported also for nonmetallic nanoparticles (Muddana et al., 2009).

- Nanoparticles: FLIM helps identify and localise nanoparticles in biological tissue via their long luminescence decay times (Becker et al., 2011b) or their second-harmonic generation (SHG) emission (Masters \& So, 2008). This is important when such particles are used as carriers of drug delivery (Prow et al., 2011), or when the diffusion of nanoparticles through skin has to be examined (Lin et al., 2011).

\section{Measurement of $\mathrm{pH}$ and Ion concentrations}

An example of FLIM-based $\mathrm{pH}$ measurement is shown in Figure 7 (left). A skin sample was stained with BCECF $\left(2^{\prime}, 7^{\prime}\right.$-bis(2-carboxyethyl)-5-(and-6)-carboxyfluorescein), and a FLIM image taken. BCECF has a protonated and a deprotonated form. These forms have different fluorescence lifetimes. The equilibrium between the forms depends on the $\mathrm{pH}$, and so does the apparent fluorescence lifetime (Hanson et al., 2002).

An example of $\mathrm{Cl}^{-}$concentration measurement is shown in Figure 7 (right). The image shows a spinal ganglion of a mouse. 6-Methoxy-quinolyl acetoethyl ester (MQAE) was used as a fluorescent probe. MQAE is quenched by $\mathrm{Cl}^{-}$, thus short lifetime means high $\mathrm{Cl}^{-}$concentration. Details were described by Kaneko et al. (2004). By using two-photon excitation and
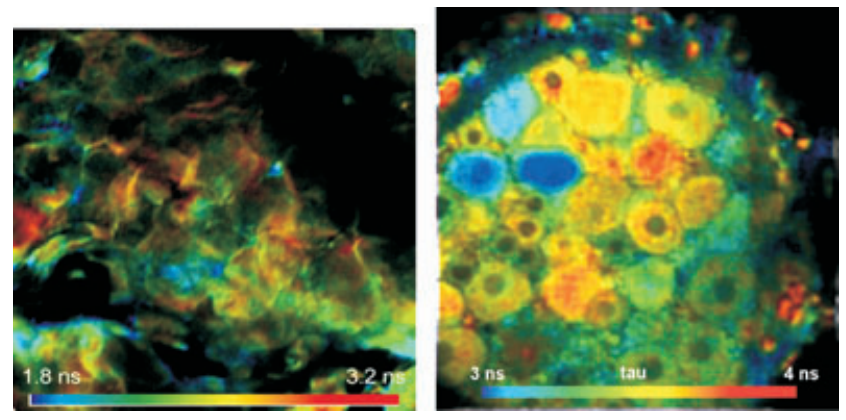

Fig. 7. Left: Lifetime image of skin tissue stained with BCECF. The lifetime is an indicator of the $\mathrm{pH}$. (Theodora Mauro, University of San Francisco, Zeiss LSM 510 NLO with bh SPC-830). Right: Spinal ganglion of a mouse stained with MQAE. Short lifetime indicates high Cl- concentration. Data courtesy of Thomas Gensch, Jülich Research Centre, Germany.

TCSPCFLIM, the authors were able to obtain $z$-stacks of the $\mathrm{Cl}^{-}$ concentration in dendrites over depth intervals up to $150 \mu \mathrm{m}$. Changes in the $\mathrm{Cl}^{-}$concentration during chloride homeostasis in neurons were investigated by Gilbert et al., (2007), changes on inflammation by Funk et al. (2008).

Due to the large lifetime shifts, FLIM measurements of $\mathrm{pH}$ and ion concentrations are relatively easy as long as only relative concentration changes are considered. Absolute measurements are far more difficult. The fluorescence lifetimes of the dye in the biological environment is not necessarily the same as in aqueous solution. Therefore, calibrations in the expected biological environment are required, which are not easy to obtain.

\section{FRET}

FRET is an interaction of two fluorophore molecules with the emission band of one overlapping the absorption band of the other. In this case energy from the first dye, the donor, can transfer immediately to the second one, the acceptor. The energy transfer is a dipole-dipole interaction and does not involve any light emission and absorption (Förster, 1946, 1948, see also Förster 2012, translation by Klaus Suhling). The principle is shown schematically in Figure 8 (left). The energy transfer rate from the donor to the acceptor decreases with the sixth power of the distance. For organic fluorophores it is noticeable only at distances shorter than $10 \mathrm{~nm}$ (Lakowicz, 2006). FRET results in a quenching of the donor fluorescence and consequently, decrease in the donor lifetime, see Figure 8 (right).

Because of its dependence on the distance on the molecular scale FRET has become an important tool of cell biology (Patterson et al., 2000; Periasamy, 2001; Hink et al., 2003; Periasamy \& Clegg, 2009). Different proteins are labelled with the donor and the acceptor; FRET is then used to verify whether the proteins are physically linked and to determine distances on the nanometre scale. 

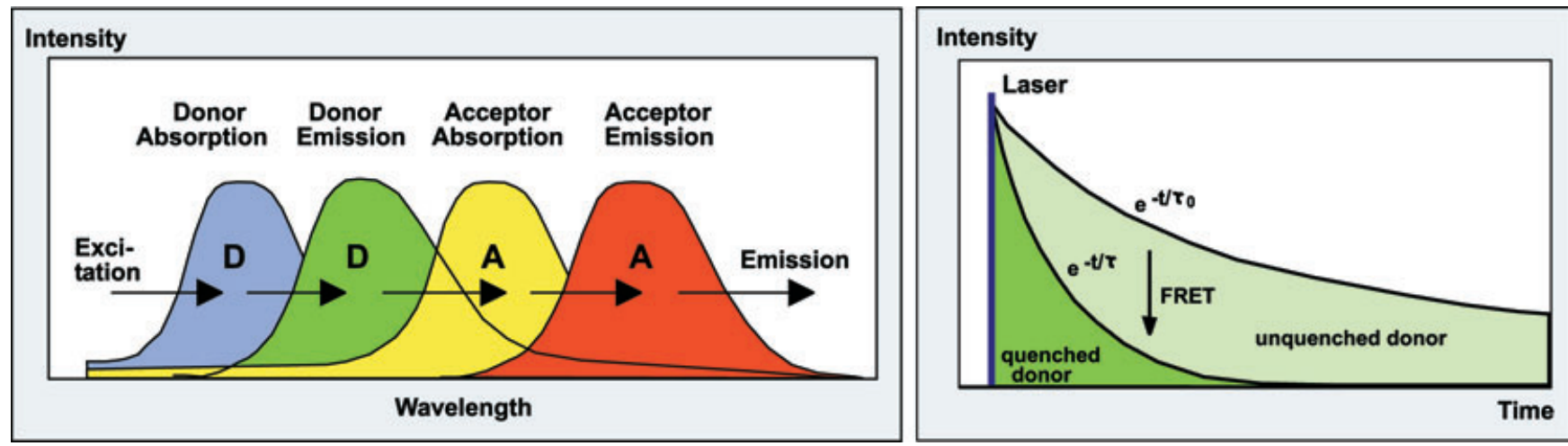

Fig. 8. Fluorescence resonance energy transfer. Left: Principle. Right: Donor decay function.

\section{Steady-state FRET measurement}

Steady state (intensity-based) FRET measurements have to take into account that the fluorescence intensities of the donor and the acceptor depend on the concentration. The 'FRET efficiency' must therefore be derived from the intensity ratio of the donor and the acceptor fluorescence. The problem is that the FRET-excited acceptor intensity is not directly available. There is 'donor bleedthrough' due to the overlap of the donor fluorescence into the acceptor emission band and there is a contribution from directly excited acceptor molecules. Steadystate FRET techniques therefore require careful calibration of these effects, using data of samples containing only the donor and only the acceptor (Gordon et al., 1998).

Another problem is the stoichiometry of the labelling. Ideally, each donor target and each acceptor target should be labelled with exactly one donor and acceptor. This can rarely be achieved in practice. However, if the acceptor labelling is incomplete the FRET efficiency obtained does not represent the distance of donor and acceptor. If the acceptor gets overlabelled correcting for directly excited acceptor fluorescence becomes difficult. It may even happen that donors interact with several acceptors. This increases the FRET efficiency which, again, does not represent the distance of donor and acceptor.

FRET measurement based on the fluorescence lifetime

FLIM-FRET techniques are based on the measurement of the fluorescence lifetime of the donor. The donor fluorescence can be obtained without contamination from the acceptor. Therefore the bleedthrough problems are intrinsically avoided (Chen \& Periasamy, 2004). The FRET efficiency is obtained by comparing the fluorescence lifetimes of the donor in presence and in absence of FRET.

An example is shown in Figure 9. The decrease in the fluorescence lifetime within the regions where FRET occurs is clearly visible, see decay curves on the right.
FRET measurement based on double-exponential decay analysis

The (probably most significant) advantage of FLIM-based FRET measurement is that FLIM can distinguish between interacting and noninteracting donor fractions. There are various reasons why a donor molecule may not interact with an acceptor. The trivial one is that some of the donor molecules are not correctly linked to their targets, or that not all acceptor targets contain the acceptor. Another reason is that the relative orientation of donor and acceptor is random. Donor molecules perpendicular to the acceptor do not interact. The problem is usually addressed by introducing the ' $\kappa^{2}$ factor' in the calculation of the FRET efficiency (Lakowicz, 2006).

More important for protein-interaction experiments is that there is usually a mixture of interacting and noninteracting proteins. Both the fraction of interacting proteins and the distance between the proteins influence the classic 'FRET efficiency'. It therefore does not tell whether a variation in the FRET efficiency is due to a variation in the distance between donor and acceptor or in the fraction of interacting proteins.

TCSPC FLIM solves the problem by double-exponential decay analysis. The donor decay functions are approximated by a double-exponential model, with a slow lifetime component from the noninteracting (unquenched) and a fast component from the interacting (quenched) donor molecules. If the labelling is complete, as can be expected for cells expressing fusion proteins of the GFP variants, the decay components (corrected by the $\kappa^{2}$ factor) represent the fractions of interacting and noninteracting proteins. The composition of the donor decay function is illustrated in Figure 10.

Double-exponential decay analysis delivers the lifetimes, $\tau_{0}$ and $\tau_{\text {fret }}$, and the amplitudes, $a_{1}$ and $a_{2}$, of the two decay components. From these parameters can be derived the true FRET efficiency for the interacting proteins, the ratio of the distance and the Förster radius, and the ratio of the relative numbers of interacting and noninteracting donor molecules. Please note that a reference lifetime from a donor-only cell is no longer required. The reference lifetime is the lifetime, $\tau_{0}$, of the 

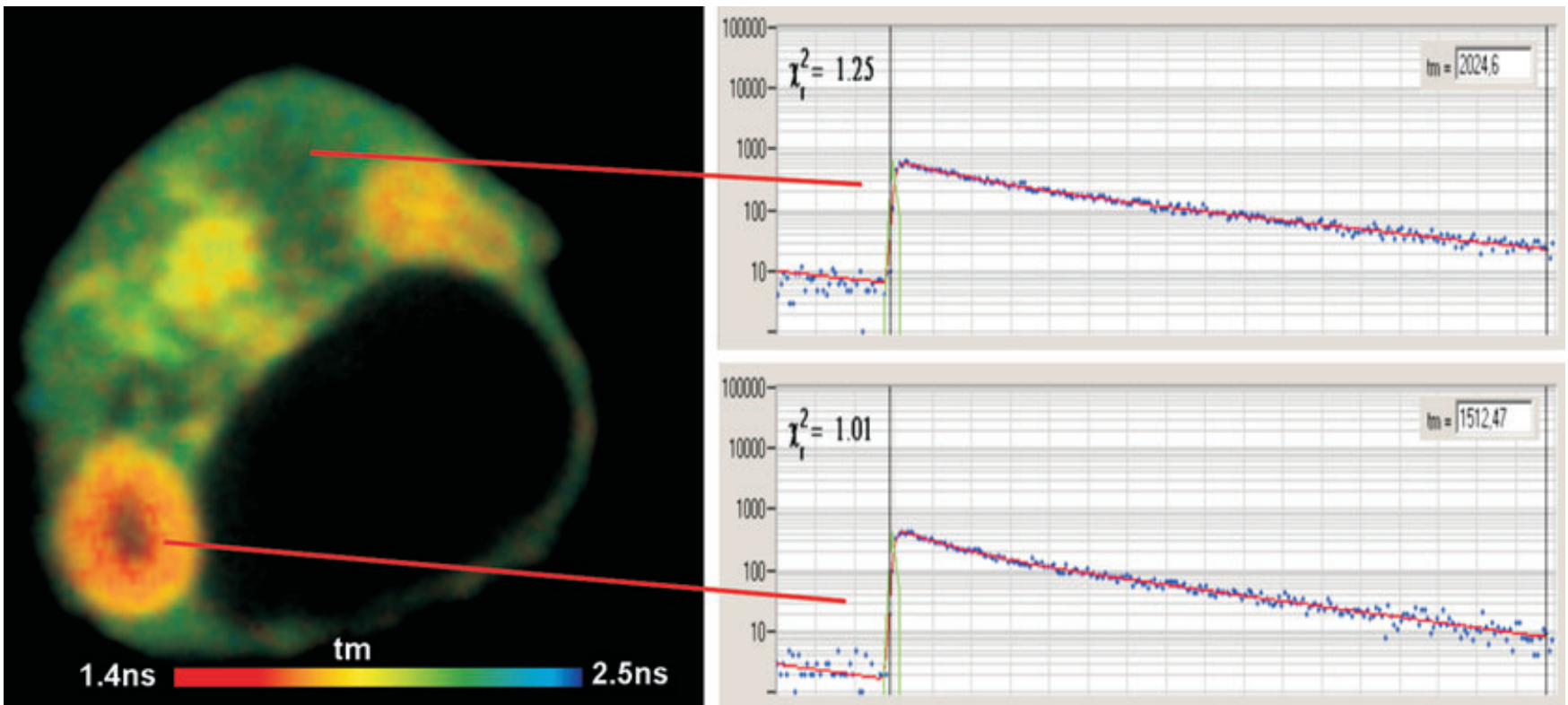

Fig. 9. FRET in an HEK cell. Interacting proteins were labelled with CFP (donor) and YFP (acceptor). Donor lifetime image, amplitude-weighted lifetime of double-exponential model. Right: Decay curves in indicated regions of the image. (Data Courtesy of Christoph Biskup, University Jena, Germany).

slow donor decay component. The advantage is that $\tau_{0}$ comes from the same local environment as $\tau_{\text {fret }}$. Double-exponential FRET is thus, within reasonable limits, independent of lifetime variations induced by variation in the local environment.

Figure 11 shows an example of double-exponential analysis for the cell shown in Figure 9. The left image displays the ratio of the lifetimes of the noninteracting and interacting donor fractions, $\tau_{0} / \tau_{\text {fret }}$. The distribution of $\tau_{0} / \tau_{\text {fret }}$ in different regions is shown far left. The locations of the maxima differ by only $10 \%$, corresponding to a distance variation of only $2 \%$. However, the variation in the intensity coefficients, $a_{1} / a_{2}$, is on the order of 10:1. The results show clearly that the variation in the single-exponential lifetime (Fig. 9) is almost entirely caused by a variation in the fraction of interacting proteins, not by a change in distance. In other words, interpreting variations in the single-exponential lifetime (or classic FRET efficiencies from steady-state experiments) as distance variations can lead to wrong results.

Double-exponential decay behaviour is commonly found in FRET experiments based on multidimensional TCSPC (Bacskai et al., 2003; Calleja et al., 2003; Becker et al., 2004a; Biskup et al., 2004b; Duncan et al., 2004; Peter \& Ameer-Beg, 2004; Ellis et al., 2008). Double-exponential decay profiles have also been confirmed by streak-camera measurements (Biskup et al., 2004a). There is no doubt that the double-exponential

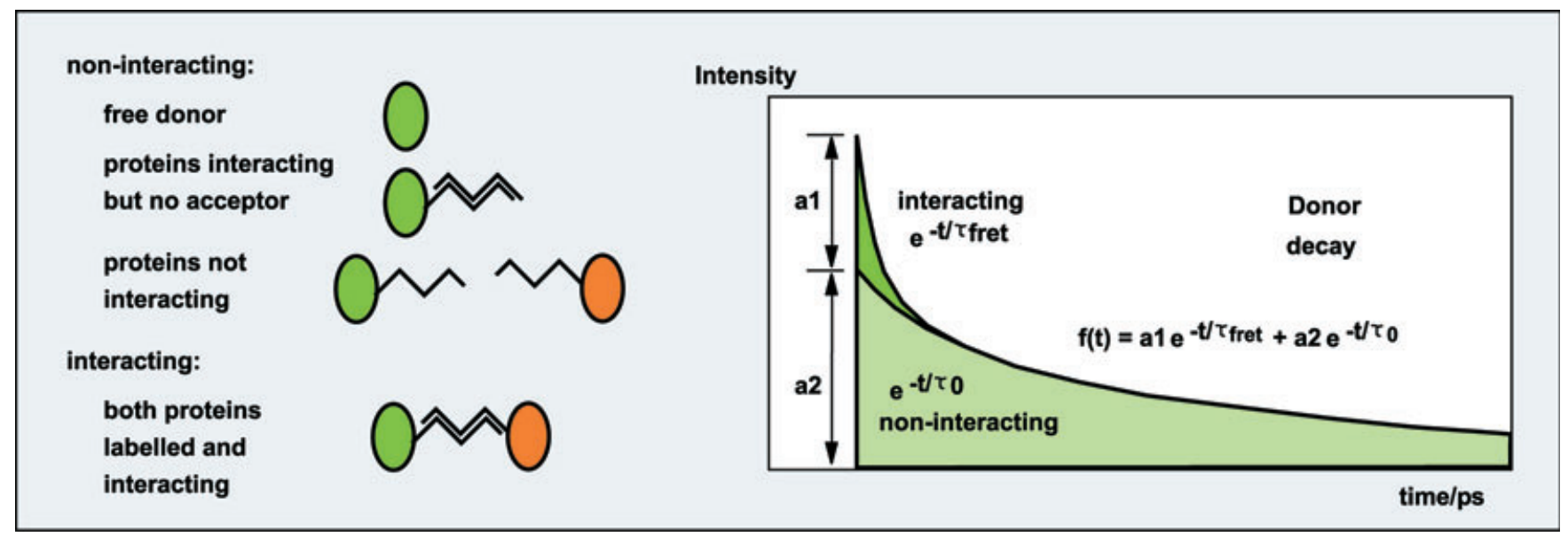

Fig. 10. Fluorescence decay components in FRET systems. 

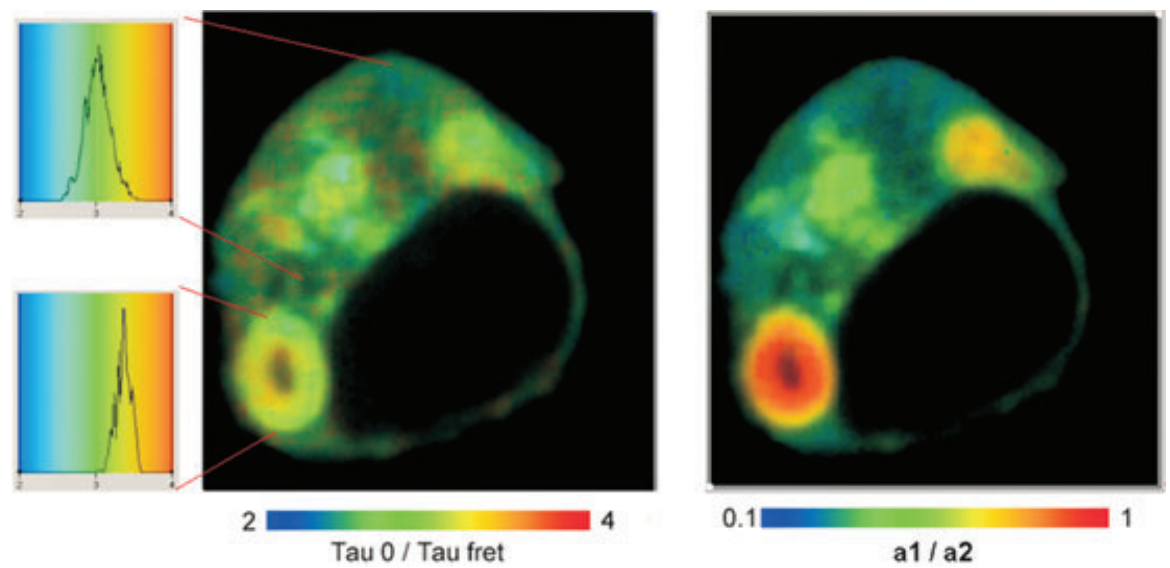

Fig. 11. FRET results obtained by double exponential lifetime analysis. Left: $\tau_{0} / \tau_{\text {fret }}$, Right: amplitude ratio, $a_{1} / a_{2}$, of interacting and noninteracting donor fractions.

decay profiles are due to the presence of an interacting and a noninteracting donor fraction.

The double-exponential decay profiles have an impact on the calculation of conventional (steady-state) FRET efficiencies from FLIM data. A double-exponential decay cannot be described by a single 'fluorescence lifetime'. To obtain the correct energy transfer efficiency an amplitudeweighted average of the two lifetime components has to be used (Lakowicz, 2006). The amplitude-weighted average is different from the lifetime obtained from a single-exponential fit. Thus, correct (conventional) FRET efficiencies are only obtained by double-exponential decay analysis.

It should be noted that there are a few more pitfalls of FRET experiments: The stoichiometry may be wrong, proteins labelled with a donor may not have their counterparts labelled with an acceptor, or the acceptor is overlabelled so that the donor interacts with several acceptors (Vogel et al., 2006; Koushik \& Vogel, 2008). The donor itself may have a multiexponential decay function, the donor signal may be contaminated by autofluorescence, or photobleaching may convert a part of the donor during the measurement (Hoffmann et al., 2008). Moreover, problems can occur if FRET measurement is attempted in fixed cells. Fixation not only changes the protein conformation but also induces unpredictable lifetime changes and multiexponential decay behaviour in the donor (Becker, 2010). Although FLIM does not necessarily solve such problems it usually helps to identify the source of the problem.

An enormous amount of FLIM-FRET papers have been published in the past few years. Please see Becker (2010) and Lakowicz (2006) for more references.

\section{Autofluorescence}

The fluorescence decay times of most endogenous fluorophores (König \& Riemann, 2003; Richards-Kortum et al., 2003; Schweitzer et al., 2007) depend on the binding to proteins, the metabolic state of the tissue, the oxygen concentration, and other biologically relevant parameters. The decay functions of autofluorescence therefore contain information about the metabolic state and the constitution of the tissue.

Due to the presence of several fluorophores or fluorophore fractions the fluorescence decay profiles of tissue autofluorescence are multiexponential, with decay components from about 100 ps to several ns. In FLIM data obtained with multiphoton microscopes also contribution from SHG may be present (Masters \& So, 2008). Because SGH is an ultrafast process it shows up as an infinitely fast decay component.

The deviations from single-exponential decay are substantial, see Figure 13. Extracting meaningful decay parameters from the data therefore requires recording at high time resolution, sufficient number of time channels, and analysis by double-exponential or triple-exponential decay models.

Especially interesting to clinical imaging are the fluorescence signals from NADH and FAD. It is known that the fluorescence lifetimes of NADH and FAD depend on the binding to proteins (Lakowicz et al., 1992; Paul \& Schneckenburger, 1996). The ratio of bound and unbound NADH depends on the metabolic state (Bird et al., 2005; Chorvat \& Chorvatova, 2006, 2009; Chia et al., 2008; Guo et al., 2008; Wang et al., 2008), and the intensity ratio of the NADH and FAD fluorescence depends on the redox state (Chance, 1976; Chance et al., 1979).

FLIM data of NADH and FAD have been used to detect precancerous and cancerous alterations (Bird et al., 2005; Kantelhardt et al., 2007; Leppert et al., 2006; Skala et al., 2007a, b; Provenzano et al., 2008). Changes in the fluorescence decay parameters are also found for various skin lesions (König \& Riemann, 2003; Dimitrow et al., 2009; 

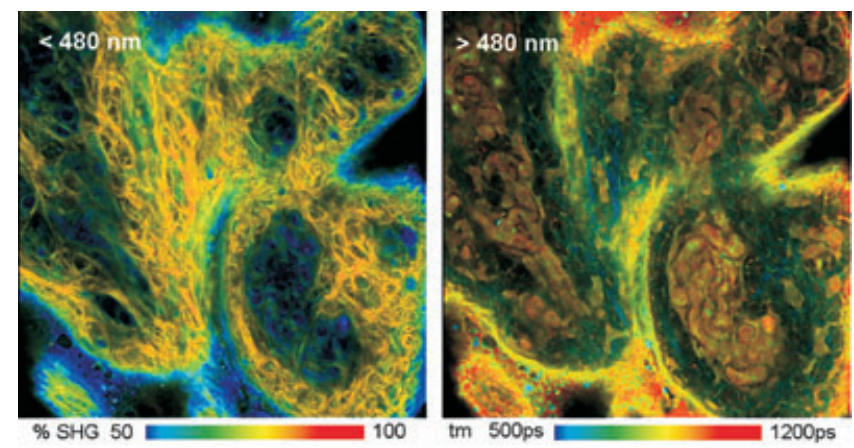

Fig. 12. Two-photon FLIM of pig skin. Two-photon excitation, nondescanned detection. Left: Wavelength channel $<480 \mathrm{~nm}$, colour shows percentage of SHG in the recorded signal. Right: Wavelength channel $>480 \mathrm{~nm}$, colour shows amplitude-weighted mean lifetime. Microscope Zeiss LSM 710 NLO, with bh Simple-Tau 152 FLIM dualchannel FLIM system. From Becker et al. (2011a).

König \& Uchugonova, 2009) and for various diseases of the fundus of the human eye (Schweitzer et al., 2004, 2007, 2009, 2010).

It has also been shown that the fluorescence decay parameters of the NADH fluorescence change with maturation of cells, during differentiation of stem cells, and during apoptosis and necrosis (Guo et al., 2008; Ghukassian \& Kao, 2009; König \& Uchugonova, 2009; Sanchez et al., 2010; König et al., 2011). A few examples of autofluorescencelifetime images are shown in Figures 12- 16.

Figures 12 and 14 give an impression of how rich in detail tissue autofluorescence FLIM data can be. The images show autofluorescence data recorded at a pig skin sample exited by two-photon excitation at $800 \mathrm{~nm}$. A Zeiss LSM 710 NLO multiphoton microscope (Carl Zeiss MicroImaging GmbH, Jena, Germany) with nondescanned detection was used. Signals in two wavelength ranges were recorded simultaneously by the two parallel channels of a Becker \& Hickl Simple-Tau 152 TCSPC FLIM system (Becker \& Hickl GmbH, Berlin, Germany). The left image shows the wavelength channel below $480 \mathrm{~nm}$. This channel contains both fluorescence and SHG signals. The intensity ratio of fluorescence and SHG is an indicator of the state of the skin (Koehler et al., 2006). The SHG component was therefore extracted from the FLIM data and its relative intensity displayed by colour. The right image is from the channel
$>480 \mathrm{~nm}$. It contains only fluorescence. It was analysed by a double-exponential decay model; the colour represents the amplitude-weighted mean lifetime. The lifetime of the fast decay component, the amplitude of the fast decay component, and the lifetime of the slow decay component are shown in Figure 14.

Figure 15 shows a lifetime image of live stem cells. The cells were excited by two-photon excitation at $750 \mathrm{~nm}$; the fluorescence was detected from 400 to $480 \mathrm{~nm}$. The emission in this interval is dominated by NADH fluorescence. As can be seen from the figure, differentiated cells have a lower $a_{1} / a_{2}$ amplitude ratio and longer amplitude-weighted lifetime.

Images of mouse glioma cells in a mouse brain are shown in Figure 16. The lifetimes of the glioma cells are longer than those of healthy cells.

\section{Concluding remarks}

It should not be concealed that FLIM, as any highend research technique, has to deal with a number of problems. The most significant one is photobleaching. At first glance, photobleaching in FLIM experiments may appear insignificant: FLIM is independent of the concentration. If a part of the fluorophores is photobleached the intensity decreases but the remaining fluorophore still emits with the correct fluorescence lifetime. The problem is, however, that there is usually a number of different fluorophores, or fluorophore fractions in different states of interaction with the environment. These photobleach at different rate, and the composition of the fluorescence decay changes. The problem is enhanced by the fact that FLIM needs to record a large number of photons. This may look surprising: For a given number of photons recorded, the signal-to-noise ratio of the fluorescence lifetime is the same as that of the intensity (Gerritsen et al., 2002). The difference is that an intensity image with, for example, $10 \%$ noise displays the spatial structures of a sample very well. However, lifetime variations to be recorded by FLIM are usually smaller than $10 \%$. Consequently, more photons are required. The problems are enhanced by the fact that the fluorophores in typical FLIM applications are linked to highly specific targets in the cells. That implies that the fluorophore concentration is low. For a given number of photons the molecules have to perform more excitation-emission cycles and, consequently, photobleach faster.
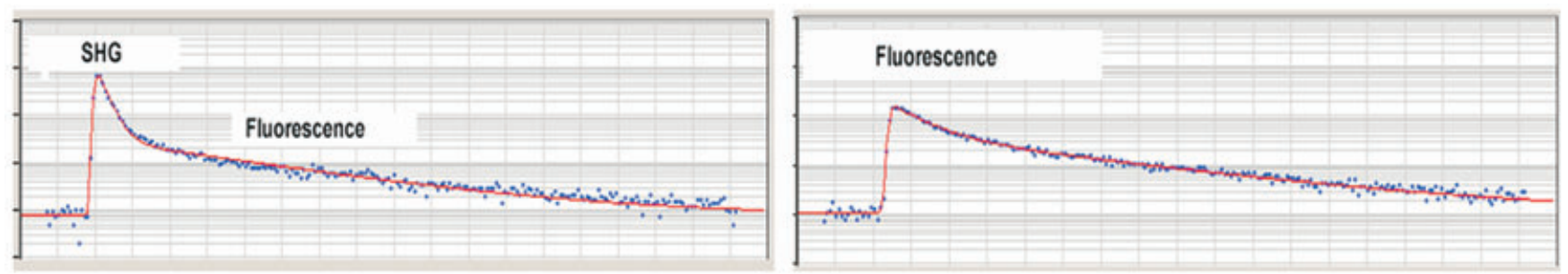

Fig. 13. Decay curves of a $3 \times 3$ pixel area in the centre of the images shown in Fig. 12 . 


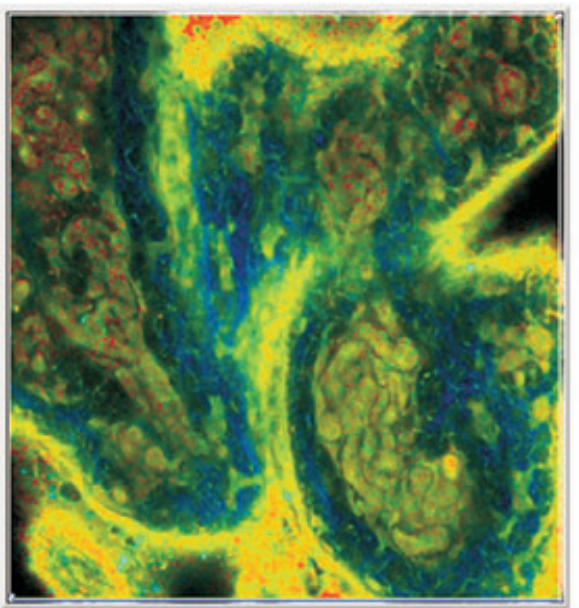

t1 100ps

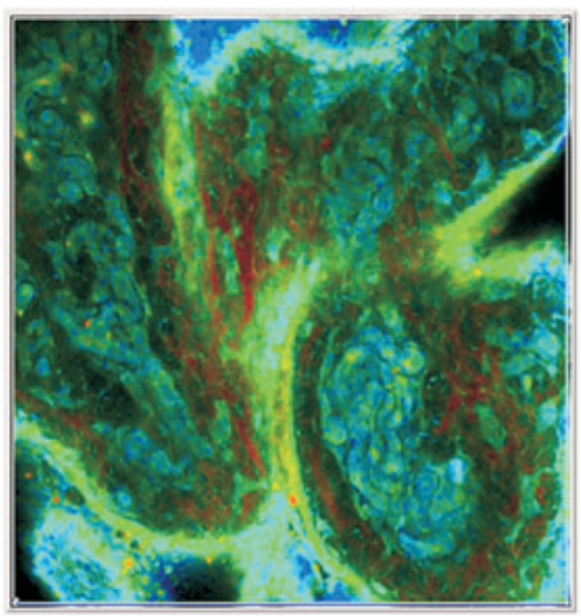

a1 0.6

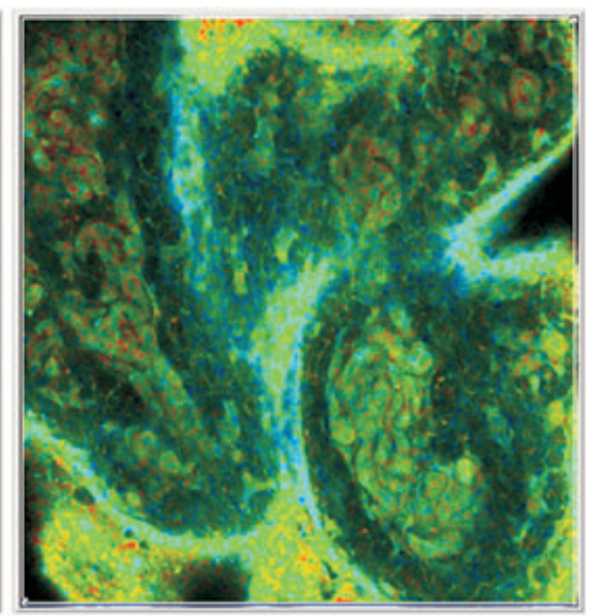

2. 2ns

Fig. 14. Wavelength channel $>480 \mathrm{~nm}$, images of the lifetime of the fast decay component, $t_{1}$, the amplitude of the fast decay component, $a_{1}$, and the lifetime of the slow decay component, $t_{2}$

Improvements can only be obtained by increasing the detection efficiency of a FLIM system. One way to do so is to use the right optical system. Nondescanned detection paths of multiphoton microscopes are often surprisingly inefficient in transmitting scattered photons. Also, efficiency is often given away by using microscope lenses of unnecessarily low numerical aperture. Another critical element is the detector. Earlier FLIM systems used high-speed photomultipliers and image intensifiers with conventional photocathodes. These rarely had a detection efficiency of more than $15 \%$. Higher efficiency is obtained by single-photon-avalanche photodiodes. However, these have extremely small areas, and thus cannot be used in nondescanned detection systems of multiphoton microscopes. A breakthrough has been achieved by the introduction of hybrid detectors with GaAsP cathodes
(Becker et al., 2011a). With these detectors photobleaching is no longer a serious problem. Even Z-stack FLIM of tissue autofluorescence has become possible.

Although there is an increasing number of papers based on FLIM the full potential of FLIM has not been exploited yet. Most fluorophores used in microscopy show lifetime variations depending on their binding targets. However, very little has been done to test these fluorophores as probes for biologically relevant parameters. In FRET applications, FLIM can work with smaller spectral separation of donor and acceptor fluorescence. It is even possible to use nonfluorescent acceptors. This could result in new donor-acceptor pairs with substantially higher FRET efficiency. Moreover, FLIM may open new ways in medical diagnostics. Applications of FLIM in dermatology (König, 2008; Roberts et al., 2011) and
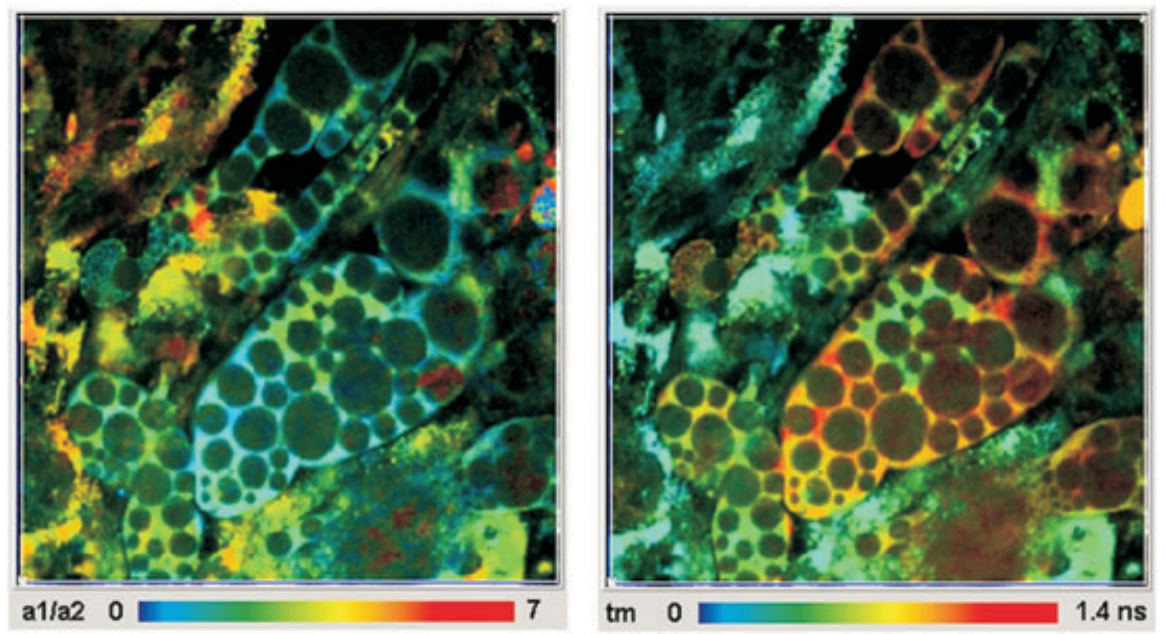

Fig. 15. FLIM of human salivary gland stem cells. Differentiated cells have significantly lower $a_{1} / a_{2}$ ratio and longer mean lifetime. Data courtesy of Aisada Uchugonova and Karsten König, Saarland University, Saarbruecken. 


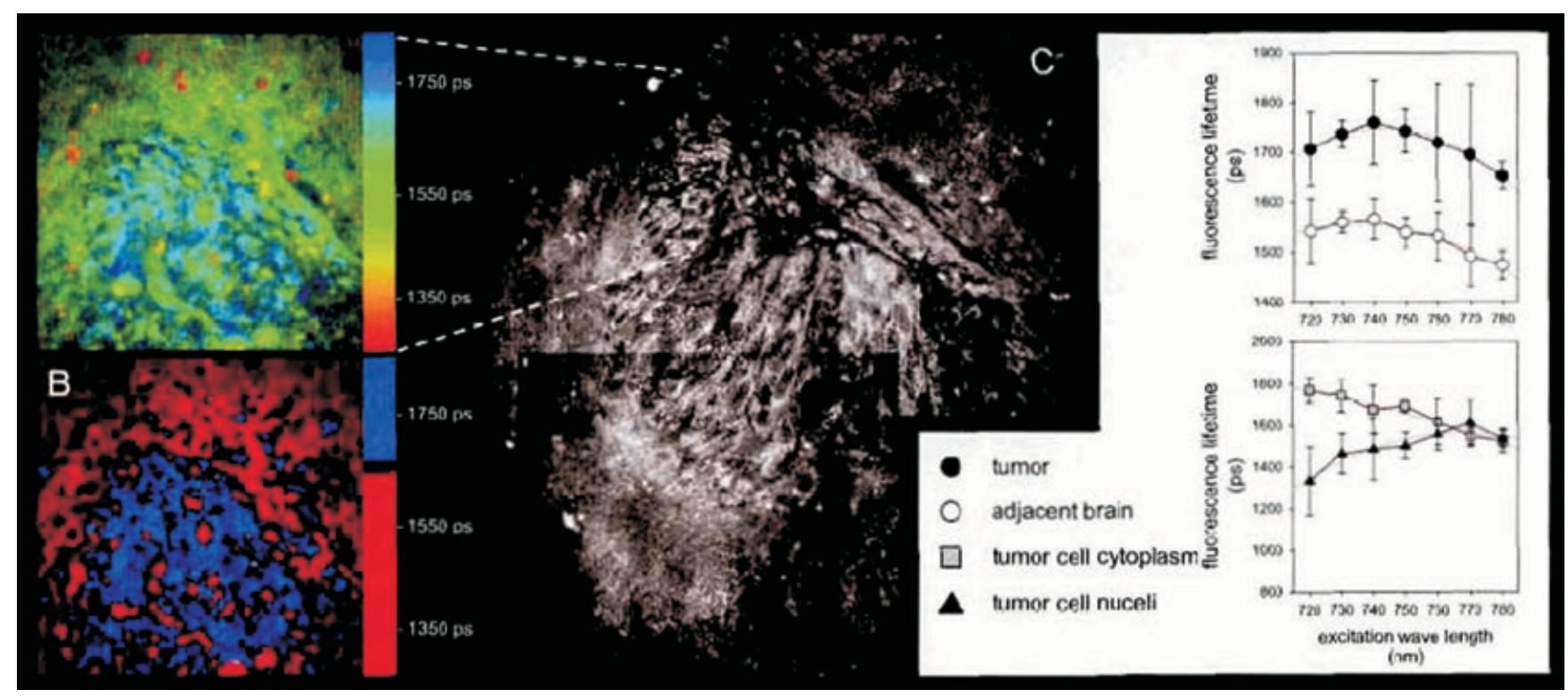

Fig. 16. Lifetime images of glioma cells in mouse brain. (A) Lifetime image, continuous colour scale. (B) Lifetime image, discrete colour scale. (C) Intensity images. Right: Lifetimes of selected cells for different excitation wavelength. From Kantelhardt et al. (2007).

ophthalmology (Schweitzer et al., 2009, 2010) are in the state of clinical trials. The first clinical FLIM systems are already on the market or close to market introduction (König, 2008; Schweitzer et al., 2009). There are also clinical applications that use diffuse optical (photon migration) techniques. These techniques work in the near infrared range. Consequently, there is an increasing interest in NIR dyes and their behaviour on the cell level. FLIM microscopy in the NIR may therefore come up soon.

An interesting question is whether FLIM can be combined with super-resolution microscopy techniques like stochastic optical reconstruction microscopy (STORM), photoactivated localisation microscopy (PALM), stimulated emission-depletion microscopy (STED), near-field scanning optical microscope (NSOM), or total internal reflection (TIRF). STORM and (PALM) require switchable fluorophores. Whether these deliver any biological information via their fluorescence lifetimes is not known. Also, the techniques require wide-field detectors with extremely high pixel numbers. FLIM applications for STORM and PALM probably have to wait until more switchable fluoprophores and suitable time-resolved detectors are available. STED uses a scanning process and single-point detection. It can be combined with FLIM as has been shown by Auksorius et al. (2008). NSOM techniques are also based on scanning. The combination with FLIM is straightforward, results have been published by Cadby et al. (2005). TIRF can be obtained both by wide-field excitation and detection and by single-point scanning. Both variants can be combined with appropriate FLIM techniques.

Biological systems often show fast dynamic effects due to metabolic activity. Such effects can be investigated by recording fast FLIM time series. However, as the recording speed is increased, a photon-budget problem develops. Improvements can be expected from techniques that use repetitive stimulation of the sample and triggered accumulation of a time series. An example for TCSPC is fluorescence lifetime-transient scanning (Becker et al., 2012), but similar principles can be applied to other FLIM techniques as well. Another, yet data-intensive, way to record a fast time series of FLIM data, and to apply correlation analysis to the temporal data of the series. It is then not required that each individual step of the series has enough photons to calculate a reasonable lifetime image.

Rotational and lateral diffusion processes in cells can be recorded by anisotropy decay imaging and fluorescence recovery after photobleaching (FRAP). Anisotropy FLIM can be done in any dual-channel FLIM system. All that is required is a polarising beamsplitter that separates the fluorescence components parallel and perpendicular to the polarisation of the excitation (Levitt et al., 2009b). FRAP can be combined with FLIM by recording a simple time series after photobleaching a region of the sample. Whether fluorescence-lifetime recording delivers useful information in FRAP experiments has to be explored yet.

\section{References}

Agronskaia, A.V., Tertoolen, L. \& Gerritsen, H.C. (2003) High frame rate fluorescence lifetime imaging. J. Phys. D: Appl. Phys. 36, 1655-1622. Auksorius, E., Boruah, B.R., Dunsby, C., Lanigan, P.M.P., Kennedy, G., Neil, M.A.A. \& French, P.M.W. (2008) Stimulated emission depletion 
microscopy with a supercontinuum source and fluorescence lifetime imaging. Opt. Lett. 33, 113-115.

Bacskai, B.J., Skoch, J., Hickey, G.A., Allen, R. \& Hyman, B.T. (2003) Fluorescence resonance energy transfer determinations using multiphoton fluorescence lifetime imaging microscopy to characterize amyloid-beta plaques. J. Biomed. Opt. 8, 368-375.

Ballew, R.M. \& Demas, J.N. (1989) An error analysis of the rapid lifetime determination method for the evaluation of single exponential decays. Anal. Chem. 61, 30-33.

Becker, W. (2005) Advanced Time-Correlated Single-Photon Counting Techniques. Springer, Berlin/Heidelberg/New York.

Becker, W. (2010) The bh TCSPC Handbook, 4th edn. Becker \& Hickl GmbH, Berlin at available on www.becker-hickl.com.

Becker, W.\&Bergmann, A. (2008) Lifetime-resolved imaging in nonlinear microscopy. In: Handbook of Biomedical Nonlinear Optical Microscopy (ed. by B.R. Masters \& P.T.C So), Oxford University Press, Oxford, NY, pp. 499-556.

Becker, W., Bergmann, A., Biskup, C., Zimmer, T., Klöcker, N. \& Benndorf, K. (2002) Multi-wavelength TCSPC lifetime imaging. Proc. SPIE 4620, $79-84$.

Becker, W., Bergmann, A., Hink, M.A., König, K., Benndorf, K. \& Biskup, C. (2004a) Fluorescence lifetime imaging by time-correlated single photon counting. Microsc. Res. Tech. 63, 58-66.

Becker, W., Bergmann, A., Biscotti, G., König, K., Riemann I., Kelbauskas, L. \& Biskup, C. (2004b) High-speed FLIM data acquisition by timecorrelated single photon counting. Proc. SPIE 5323, 27-35.

Becker, W., Bergmann, A., Haustein, E. et al. (2006) Fluorescence lifetime images and correlation spectra obtained by multi-dimensional TCSPC. Microsc. Res. Tech. 69, 186-195.

Becker, W., Bergmann, A. \& Biskup, C. (2007) Multi-spectral fluorescence lifetime imaging by TCSPC. Microsc. Res. Tech. 70, 403-409.

Becker, W., Su, B. \& Bergmann, A. (2009) Fast-acquisition multispectral FLIM by parallel TCSPC. Proc. SPIE 7183, 7183051-5.

Becker, W., Su, B., Weisshart, K. \& Holub, O. (2011a) FLIM and FCS detection in laser-scanning microscopes: increased efficiency by GaAsP hybrid detectors. Microsc. Res. Tech. 74, 804-811.

Becker, W., Su, B., Bergmann, A., Weisshart, K. \& Holub, O. (2011b) Simultaneous fluorescence and phosphorescence lifetime imaging. Proc. SPIE 7903, 790320-1-7.

Becker, W., Su, B. \& Bergmann, A. (2012) Spatially resolved recording of transient fluorescence-lifetime effects by line-scanning TCSPC. Proc. SPIE 8226, 82260C-1-6.

Benesch, J., Hungerford, G., Suhling, K., Tregidgo, C., Mano, J.F. \& Reis, R.L. (2007) Fluorescence probe techniques to monitor protein adsorption-induced conformation changes on biodegradable polymers. J. Colloid Interface Sci. 312, 193-200.

Berezin, M.Y. \& Achilefu, S. (2010) Fluorescence lifetime maesurement and biological imaging. Chem. Rev. 110, 2641-2684.

Bird, D.K., Eliceiri, K.W., Fan, C-H. \& White, J.G. (2004) Simultaneous two-photon spectral and lifetime fluorescence microscopy. Appl. Opt. 43, 5173-5182.

Bird, D.K., Yan, L., Vrotsos, K.M., Eliceiri, K. E. \& Vaughan, E. M. (2005) Metabolic mapping of MCF10A human breast cells via multiphoton fluorescence lifetime imaging of coenzyme NADH. Cancer Res. 65, 8766-8773.

Biskup, C., Zimmer, T. \& Benndorf, K. (2004a) FRET between cardiac $\mathrm{Na}^{+}$ channel subunits measured with a confocal microscope and a streak camera. Nat. Biotechnol. 22, 220-224.
Biskup, C., Kelbauskas, L., Zimmer, T., et al. (2004b) Interaction of PSD95 with potassium channels visualized by fluorescence lifetime-based resonance energy transfer imaging. J. Biomed. Opt. 9, 735-759.

Biskup, C., Zimmer, T., Kelbauskas, L., Hoffmann, B., Klöcker, N., Becker, W., Bergmann, A. \& Benndorf, K. (2007) Multi-dimensional fluorescence lifetime and FRET measurements. Microsc. Res. Tech. 70, 403-409.

Booth, M.J. \&Wilson, T. (2004) Low-cost, frequency-domain, fluorescence lifetime confocal microscopy. J. Microsc. 214, 36-42.

Buurman, E.P., Sanders, R., Draaijer, A., Gerritsen, H.C., van Veen, J.J.F., Houpt, P.M. \& Levine, Y.K. (1992) Fluorescence lifetime imaging using a confocal laser scanning microscope. Scanning 14, 155-159.

Cadby, A., Dean, R., Fox, A.M., Jones, R.A.L., \& Lidzey D.G. (2005) Mapping the fluorescence decay lifetime of a conjugated polymer in a phase-separated blend using a scanning near-field optical microscope. Nano Lett. 5, 2232-2237.

Cade, N.I., Fruhwirth, G., Archibald, S. J., Ng, T. \& Richards, D. (2010) A cellular screening assay using analysis of metal-modified fluorescence lifetime. Biophys. J. 98, 2752-2757.

Calleja, V., Ameer-Beg, S., Vojnovic, B., Woscholski, R., Downwards, J. \& Larijani, B. (2003) Monitoring conformational changes of proteins in cells by fluorescence lifetime imaging microscopy. Biochem. J. 372, 33-40.

Chance, B. (1976) Pyridine nucleotide as an indicator of the oxygen requirements for energy-linked functions of mitochondria. Circ. Res. 38, I31-I38.

Chance, B., Schoener, B., Oshino, R., Itshak, F. \& Nakase, Y. (1979) Oxidation-reduction ratio studies of mitochondria in freeze-trapped samples. NADH and flavoprotein fluorescence signals. J. Biol. Chem. 254, 4764-4771.

Chen, Y. \& Periasamy, A. (2004) Characterization of two-photon excitation fluorescence lifetime imaging microscopy for protein localization. Microsc. Res. Tech. 63, 72-80.

Chia, T.H., Williamson, A., Spencer, D.D. \& Levene, M.J. (2008) Multiphoton fluorescence lifetime imaging of intrinsic fluorescence in human and rat brain tissue reveals spatially distinct NADH binding. Opt. Express 16, 4237-4249.

Chorvat, D. \& Chorvatova, A. (2006) Spectrally resolved time-correlated single photon counting: a novel approach for characterization of endogenous fluorescence in isolated cardiac myocytes. Eur. Biophys. J. 36, 73-83.

Chorvat, D. \& Chorvatova, A. (2009) Multi-wavelength fluorescence lifetime spectroscopy: a new approach to the study of endogenous fluorescence in living cells and tissues. Laser Phys. Lett. 6, 175-193.

Cole, M.J., Siegel, J., Webb, S.E.D., et al. (2001) Time-domain whole-field lifetime imaging with optical sectioning. J. Microsc. 203, 246-257.

De Beule, P., Owen, D.M., Manning, H.B. et al. (2007) Rapid hyperspectral fluorescence lifetime imaging. Microsc. Res. Tech. 70, 481-484.

Denk, W., Strickler, J.H., Webb, W.W.W. (1990) Two-photon laser scanning fluorescence microscopy. Science 248, 73-76.

Diaspro, A. (ed.) (2001) Confocal and Two-Photon Microscopy: Foundations, Applications and Advances. Wiley-Liss, Inc., New York.

Digman, M.A., Caiolfa, V.R., Zamai, M. \& Gratton, E. (2008) The phasor approach to fluorescence lifetime imaging analysis. Biophys. J. 94, L14L16.

Dimitrow, E., Riemann, I., Ehlers, A., Koehler, M., Norgauer, J., Elsner, P., König, K. \& Kaatz, M. (2009) Spectral fluorescence lifetime detection 
and selective melanin imaging by multiphoton laser tomography for melanoma diagnosis. Exp. Dermatol. 18, 509-515.

Dowling, K., Hyde, S.C.W., Dainty, J.C., French, P.M.W. \& Hares, J.D. (1997) 2-D fluorescence liefetime imaging using a time-gated image intensifier. Opt. Commun. 135, 27-31.

Duncan, R.R., Bergmann, A., Cousin, M.A., Apps, D.K. \& Shipston, M.J. (2004) Multi-dimensional time-correlated single-photon counting (TCSPC) fluorescence lifetime imaging microscopy (FLIM) to detect FRET in cells. J. Microsc. 215, 1-12.

Dymoke-Bradshaw, A.K.L. (1992) The impact of high-voltage pulse technology on high-speed photography. Proc. SPIE 1757, 2-6.

Ellis, J.D., Llères, D., Denegri, M., Lamond, A.I. \& Cáceres, J.F. (2008) Spatial mapping of splicing factor complexes involved in exon and intron definition. J. Cell Biol. 181, 921-934.

Elson, D.S., Munro, I., Requejo-Isidro, J. et al. (2004) Real-time timedomain fluorescence lifetime imaging including single-shot acquisition with a segmented optical image intensifier. New J. Phys. 6, 180-192.

Felekyan, S., Kühnemuth, R., Kudryavtsev, V., Sandhagen, C., Becker, W. \& Seidel, C.A.M. (2005) Full correlation from picoseconds to seconds by time-resolved and time-correlated single photon detection. Rev. Sci. Instrum. 76, 083104-1-14.

Förster, Th. (1946) Energiewanderung und Fluoreszenz. Naturwissenschaften 6, 166-175.

Förster, Th. (1948) Zwischenmolekulare Energiewanderung und Fluoreszenz. Ann. Phys. Ser. 6 2, 55-75.

Forster, Th. (2012) Energy migration and fluorescence. Translated by Klaus Suhling. J. Biomed. Opt. 7, 011002 (2012).

Funk, K., Woitecki, A., Franjic-Würtz, C., Gensch, Th., Möhrlein, F. \& Frings, S. (2008) Modulation of chloride homeostasis by inflammatory mediators in dorsal ganglion neurons. Mol. Pain 4, 32-43.

Gerritsen, H.C., Asselbergs, M.A.H., Agronskaia, A.V. \& van Sark, W.G.J.H.M. (2002) Fluorescence lifetime imaging in scanning microscopes: acquisition speed, photon economy and lifetime resolution. J. Microsc. 206, 218-224.

Ghukassian, V. \& Kao, F.-J. (2009) Monitoring cellular metabolism with fluorescence lifetime of reduced nicotinamide adenine dinucleotide. $J$. Phys. Chem. C 113, 11532-11540.

Gilbert, D., Franjic-Würtz, C., Funk, K., Gensch, Th., Frings, S. \& Möhrlen, F. (2007) Differential maturation of chloride homeostasis in primary afferent neurons of the somatosensory system. Int. J. Dev. Neurosci. 25, $479-489$.

Gordon, G.W., Berry, G., Liang, X.H., Levine, B. \& Herman, B. (1998) Quantitative fluorescence resonance energy transfer measurements using fluorescence microscopy. Biophys. J. 74, 2702-2713.

Graham, D.L., Lowe, P.N. Chalk, P.A. (2001) A method to measure the interaction of Rac/Colc 42 with their binding partners using fluorescence resonance energy transfer between mutants of green fluorescent protein. Anal. Biochem. 296, 208-217.

Gratton, E. \& Barbieri, B.B. (1986) Multifrequency phase fluorometry using pulsed sources: theory and applications. Spectroscopy 1, 28-36.

Gratton, E., Breusegem, S., Sutin, J., Ruan, Q. \& Barry, N. (2003) Fluorescence lifetime imaging for the two-photon microscope: timedomain and frequency domain methods. J. Biomed. Opt. 8, 381-390.

Guo, H.-W., Chen, C.-T., Wei, Y.-H., Lee, O.K., Gukassyan, V., Kao, F.-J. \& Wanga, H.-W. (2008) Reduced nicotinamide adenine dinucleotide fluorescence lifetime separates human mesenchymal stem cells from differentiated progenies. J. Biomed. Opt. 13 050505-1-3.
Hanson, K.M., Behne, M.J., Barry, N.P., Mauro, T.M. \& Gratton, E. (2002) Two-photon fluorescence imaging of the skin stratum corneum $\mathrm{pH}$ gradient. Biophys. J. 83, 1682-1690.

Hink, M.A., Visser N.V., Borst, J.W. van Hoek, A.\& Visser, A.J.W.G. (2003) Practical use of corrected fluorescence excitation and emission spectra of fluorescent proteins in Förster resonance energy transfer (FRET) studies. J. Fluoresc. 13(2), 1-4.

Hoffmann, B., Zimmer, T, Klöcker, N., Kelbauskas, L., König, K., Benndorf, K. \& Biskup, C. (2008) Prolonged irradiation of enhanced cyan fluorescent protein or Cerulean can invalidate Förster resonance energy transfer measurements. J. Biomed. Opt. 13, 031250-1-9.

Kaneko, H., Putzier, I., Frings, S., Kaupp, U.B., \& Gensch, Th. (2004) Chloride accumulation in mammalian olfactory sensory neurons. J. Neurosci. 24, 7931-7938.

Kantelhardt, S.R., Leppert, J., Krajewski, J., Petkus, N., Reusche, E., Tronnier, V.M., Hüttmann, G., \& Giese, A. (2007) Imaging of brain and brain tumor specimens by time-resolved multiphoton excitation microscopy ex vivo. Neuro-Onkology 9, 103-112.

Katsoulidou, V., Bergmann, A., \& Becker, W. (2007) How fast can TCSPC FLIM be made? Proc. SPIE 6771, 67710B-1-7.

Kelbauskas, L. \& Dietel, W. (2002) Internalization of aggregated photosensitizers by tumor cells: Subcellular time-resolved fluorescence spectroscopy on derivates of pyropheophorbide-a ethers and chlorin e6 under femtosecond one- and two-photon excitation. Photochem. Photobiol. 76, 686-694.

Kemnitz, K. (2001) Picosecond fluorescence lifetime imaging spectroscopy as a new tool for 3D structure determination of macromolecules in living cells, In: New Trends in Fluorescence Spectroscopy (ed. by B. Valeur \& J.C. Brochon), Springer Verlag, Berlin/Heidelberg/New York, pp. 381-410.

Koehler, M.J., König, K., Elsner, P., Bückle, R. \& Kaatz, M. (2006) In vivo assessment of human skin aging by multiphoton laser scanning tomography. Opt. Lett. 31, 2879-2881.

Köllner, M. \& Wolfrum, J. (1992) How many photons are necessary for fluorescence-lifetime measurements? Phys. Chem. Lett. 200, 199-204.

König, K. (2008) Clinical multiphoton tomography. J. Biophoton. 1, 1323.

König, K. \& Riemann, I. (2003) High-resolution multiphoton tomography of human skin with subcellular spatial resolution and picosecond time resolution. J. Biom. Opt. 8, 432-439.

König, K. \& Uchugonova, A. (2009) Multiphoton fluorescence lifetime imaging at the dawn of clinical application. In: FLIM Microscopy in Biology and Medicine (ed. by A. Periasamy \& R.M. Clegg), CRC Press Taylor \& Francis Group, Boca Raton, FL, pp. 165-187.

König, K., Uchugonova, A. \& Gorjup, E. (2011) Multiphoton fluorescence lifetime imaging of 3D-stem cell spheroids during differentaition. Microsc. Res. Tech. 74, 9-17.

Koushik, S.V. \& Vogel, S.S. (2008) Energy migration alters the fluorescence lifetime of Cerulean: implications for fluorescence lifetime imaging Forster resonance energy transfer measurements. J. Biomed. Opt. 13, 031204-1-9.

Krishnan, R.V., Saitoh, H., Terada, H., Centonze, V.E. \& Herman, B. (2003) Development of a multiphoton fluorescence lifetime imaging microscopy (FLIM) system using a streak camera. Rev. Sci. Instrum. 74, 2714-2721.

Kuchibhotla, K.V., Lattarulo, C.R., Hyman, B. \& Bacskai, B.J. (2009) Synchronous hyperactivity and intercellular calcium waves in astrocytes in Alzheimer mice. Science 323, 1211-1215. 
Kuimova, M.K., Yahioglu, G., Levitt, J.A. \& Suhling, K. (2008) Molecular rotor measures viscosity of live cells via fluorescence lifetime imaging. J. Am. Chem. Soc. 130, 6672-6673.

Kumar, S., Dunsby, C., De Beule, P.A.A. et al. (2007) Multifocal multiphoton excitation and time correlated single photon counting detection for 3-D fluorescence lifetime imaging. Opt. Expr. 15, 1254812561.

Lakowicz, J.R. (2006) Principles of Fluorescence Spectroscopy, 3rd ed. Springer, Berlin, Heidelberg, New York.

Lakowicz, J.R. \& Berndt, K. (1991) Lifetime-selective fluorescence lifetime imaging using an rf phase-sensitive camera. Rev. Sci. Instrum. 62, 1727-1734.

Lakowicz, J.R., Szmacinski, H., Nowaczyk, K. \& Johnson, M.L. (1992) Fluorescence lifetime imaging of free and protein-bound NADH. PNAS 89, 1271-1275.

Lebedev, A.Y., Cheprakov, A.V., Sakadzic, S., Boas, D.A., Wilson, D.F. \& Vinogradov, S.A. (2009) Dendritic phosphorescent probes for oxygen imaging in biological systems. Appl. Mater. Interfaces 1, 1292-1304.

Leppert, J., Krajewski, J., Kantelhardt, S.R., Schlaffer, S., Petkus, N., Reusche, E., Hüttmann, G. \& Giese, A. (2006) Multiphoton excitation of autofluorescence for microscopy of glioma tissue. Neurosurgery $\mathbf{5 8}$, $759-767$.

Levitt, J.A., Kuimova, M.K., Yahioglu, G., Chung, P.-H., Suhling, K. \& Phillips, D. (2009a) Membrane-bound molecular rotors measure viscosity in live cells via fluorescence lifetime imaging. J. Phys. Chem. C 113, 11634-11642.

Levitt, J.A., Matthews, D.R., Ameer-Beg, S.M. \& Klaus Suhling, K. (2009b) Fluorescence lifetime and polarization-resolved imaging in cell biology. Curr. Opin. Biotechnol. 20, 28-36.

Lin, L.L., Grice, J.E., Butler, M.K. et al. (2011) Time-correlated single photon counting for simultaneous monitoring of zinc oxide nanoparticles and $\mathrm{NAD}(\mathrm{P}) \mathrm{H}$ in intact and barrier-disrupted volunteer skin. Pharm Res. 28, 2920-2930.

Malicka, J., Gryczynski, I., Geddes, C.D. \& Lakowicz, J.R. (2003) Metalenhanced emission from indocyanine green: a new approach to in vivo imaging. J. Biomed. Opt. 8, 472-478.

Masters, B.R. \& So, P.T.C. (2008) Handbook of Biomedical Nonlinear Optical Microscopy. Oxford University Press, Oxford, New York.

Mitchell, A.C., Wall, J.E., Murray, J.G. \& Morgan, C.G. (2002a) Measurement of nanosecond time-resolved fluorescence with a directly gated interline CCD camera. J. Microsc. 206, 233-238.

Mitchell, A.C., Wall, J.E., Murray, J.G. \& Morgan, C.G. (2002b) Direct modulation of the effective sensitivity of a CCD detector: a new approach to time-resolved fluorescence imaging. J. Microsc. 206, 225-232.

Muddana, H.S., Morgan, T.T., Adair, J.H. \& Butler, P.J. (2009) Photophysics of $\mathrm{Cy} 3$-encapsulated calcium phosphate nanoparticles. Nano Lett. 9, 1559-1556.

O'Connor, D.V. \& Phillips, D. (1984) Time-Correlated Single Photon Counting. Academic Press, London.

Patterson, G.H., Piston, D.W., \& Barisas, B.G. (2000) Förster distances between green fluorescent protein pairs. Anal. Biochem. 284, 438-440.

Paul, R.J. \& Schneckenburger, H. (1996) Oxygen concentration and the oxidation-reduction state of yeast: determination of free/bound NADH and flavins by time-resolved spectroscopy. Naturwissenschaften $\mathbf{8 3}, 32$ 35.

Pawley, J. (2006) Handbook of Biological Confocal Microscopy. 3rd ed. Springer.
Periasamy, A. (2001) Methods in Cellular Imaging. Oxford University Press, Oxford, New York.

Periasamy, A. \& Clegg, R.M. (2009) FLIM Microscopy in Biology and Medicine. CRC Press, Taylor \& Francis Group, Boca Raton, Fl, pp. 165187.

Peter, M. \& Ameer-Beg, S.M. (2004) Imaging molecular interactions by multiphoton FLIM. Biol. Cell 96, 231-236.

Petrasek, Z. \& Suhling, K. (2010) Photon arrival timing with sub-camera exposure time resolution in wide-field time-resolved photon counting imaging. Opt. Express 18(24), 24889-24901

Philip, J.P. \& Carlsson, K. (2003) Theoretical investigation of the signalto-noise ratio in fluorescence lifetime imaging. J. Opt. Soc. Am. A20, 368-379.

Provenzano, P.P., Rueden, C.T., Trier, S.M., Yan, L., Ponik, S.M., Inman, D.R., Keely, P.J. \& Eliceiri, K.W. (2008) Nonlinear optical imaging and spectral-lifetime computational analysis of endogenous and exogenous fluorophores in breast cancer. J. Biomed. Opt. 13, 031220-1-11.

Prow, T.W., Grice, J.E., Lin, L.L. et al. (2011) Nanoparticles and microparticles for skin drug delivery. Adv. Drug. Deliv. Rev. 63, 470491.

Prummer, M., Sick, B., Renn, A. \& Wild, U.P. (2004) Multiparameter microscopy and spectroscopy for single-molecule analysis. Anal. Chem. 76, 1633-1640.

Richards-Kortum, R., Drezek, R., Sokolov, K., Pavlova, I. \& Follen, M. (2003) Survey of endogenous biological fluorophores. In: Handbook of Biomedical Fluorescence (ed. by M.A. Mycek, B.W. Pogue), pp. 237-264. Marcel Dekker Inc. New York, Basel.

Ritman-Meer, T., Cade, N.I. \& Richards, D. (2007) Spatial imaging of modifications to fluorescence lifetime and intensity by individual $\mathrm{Ag}$ nanoparticles. Appl. Phys. Lett. 91, 123122-1-3.

Roberts, M.S., Dancik, Y., Prow, T.W., et al. (2011) Non-invasive imaging of skin physiology and percutaneous penetration using 5D (space, time and anisotropy) fluorescence spectral and lifetime imaging with multiphoton and confocal microscopy. Eur. J. Pharm. Biopharm. 77, 469-488.

Rowley, M.I., Barber, P.R., Coolen, A.C.C. \& Vojnovic, B. (2011) Bayesian analysis of fluorescence lifetime imaging data. Proc. SPIE 7903, 790325-1-7.

Rück, A., Hülshoff, Ch., Kinzler, I., Becker, W. \& Steiner, R. (2007) SLIM: a new method for molecular imaging. Microsc. Res. Tech. 70, 403-409.

Sanchez, W.Y., Prow T.W., Sanchez W.H., Grice, J.E. \& Roberts, M.S. (2010) Analysis of the metabolic deterioration of ex vivo skin, from ischemic necrosis, through the imaging of intracellular $\mathrm{NAD}(\mathrm{P}) \mathrm{H}$ by multiphoton tomography and fluorescence lifetime imaging microscopy (MPT-FLIM). J. Biomed. Opt. 15, 046008$1-11$.

Sanders, R., Draaijer, A., Gerritsen, H.C., Houpt, P.M. \& Levine, Y.K. (1995) Quantitative pH imaging in cells using confocal fluorescence lifetime imaging microscopy. Anal. Biochem. 227, 302-308.

Schweitzer, D. (2009) Quantifying fundus autofluorescence. In: Fundus Autofluorescence (ed. by N. Lois \& J.V. Forrester), Lippincott Willams \& Wilkins/Wolters Kluwer Philadelphia, PA, pp. 78-95.

Schweitzer, D. (2010) Metabolic mapping. In: Medical Retina, Essential in Opthalmology ( ed. by F.G. Holz \& R.F. Spaide), Springer, NY, 107123.

Schweitzer, D., Hammer, M., Schweitzer, F., Anders, R., Doebbecke, T., Schenke, S. \& Gaillard, E.R. (2004) In vivo measurement of 
time-resolved autofluorescence at the human fundus. J. Biomed. Opt. 9, 1214-1222.

Schweitzer, D., Schenke, S., Hammer, M., Schweitzer, F., Jentsch, S., Birckner, E. \& Becker, W. (2007) Towards metabolic mapping of the human retina. Microsc. Res. Tech. 70, 403-409.

Scully, A.D., MacRoberts, A.J., Botchway, S., O'Neill, P., Parker, A.W., Ostler, R.B. \& Phillips, R.B. (1996) Development of a laser-based fluorescence microscope with subnanosecond time resolution. J. Fluoresc. 6, 119-125

Skala, M.C., Riching, K.M., Bird, D.K., Dendron-Fitzpatrick, A., Eickhoff, J., Eliceiri, K.W., Keely, P.J. \& Ramanujam, N. (2007a) In vivo multiphoton fluorescence lifetime imaging of protein-bound and free nicotinamide adenine dinucleotide in normal and precancerous epithelia. J. Biomed. Opt. 12, 02401-1-10.

Skala, M.C., Riching, K.M., Gendron-Fitzpatrick, A., Eickhoff, J., Eliceiri, K.W., White, J.G. \& Ramanujam, N. (2007b) In vivo multiphoton microscopy of NADH and FAD redox states, fluorescence lifetimes, and cellular morphology in precancerous epithelia. PNAS 104, 19494 19499.

Straub, M. \& Hell, S.W. (1998) Fluorescence lifetime three-dimensional microscopy with picosecond precision using a multifocal multiphoton microscope. Appl. Phys. Lett. 73, 1769-1771.

Treanor, B., Lanigan, P.M.P., Suhling, K. et al. (2005) Imaging fluorescence lifetime heterogeneity applied to GFP-tagged MHC protein at an immunological synapse. J. Microsc. 217, 36-43.
Van Zandvoort, M.A.M.J., de Grauw, C.J., Gerritsen, H.C., Broers, J.L.V., Egbrink, M.G.A., Ramaekers, F.C.S. \& Slaaf, D.W. (2002) Discrimination of DNA and RNA in cells by a vital fluorescent probe: lifetime imaging of SYTO13 in healthy and apoptotic cells. Cytometry $47,226-232$.

Vogel, S.S., Thaler, C. \& Koushik, S.V. (2006) Fanciful FRET. Sci. STKE 2006, re2 1-8.

Wang, X.F., Periasamy, A., Herman, B. \& Coleman, D.M. (1992) Fluorescence lifetime imaging microscopy (FLIM): instrumentation and applications. Crti. Rev. Anal. Chem. 23, 369-395.

Wang, H.-W., Ghukassyan, V., Chen, C.T., Wei, Y.H., Guo, H.W., Yu, J.S. \& Kao, F.J. (2008) Differentiation of appoptosis from necrosis by dynamic changes of reduced nicotinamide adenine dinucleotide fluorescence lifetime in live cells. J. Biomed. Opt. 13, 054011$1-9$.

Weidkamp-Peters, S., Felekyan, S., Bleckmann, A., Simon, R., Becker, W., Kühnemuth, R. \& Seidel, C.A.M. (2009) Multiparameter fluorescence image spectroscopy to study molecular interactions. Photochem. Photobiol. Sci. 8, 470-480.

Widengren, J., Kudryavtsev, V., Antonik, M., Berger, S., Gerken, M., Seidel, C.A.M. (2006) Single-molecule detection and identification of multiple species by multiparameter fluorescence detection. Anal.Chem. 78, 2039-2050.

Wilson, T. \& Sheppard, C. (1984) Theory and Practice of Scanning Optical Microscopy. Academic Press, London. 